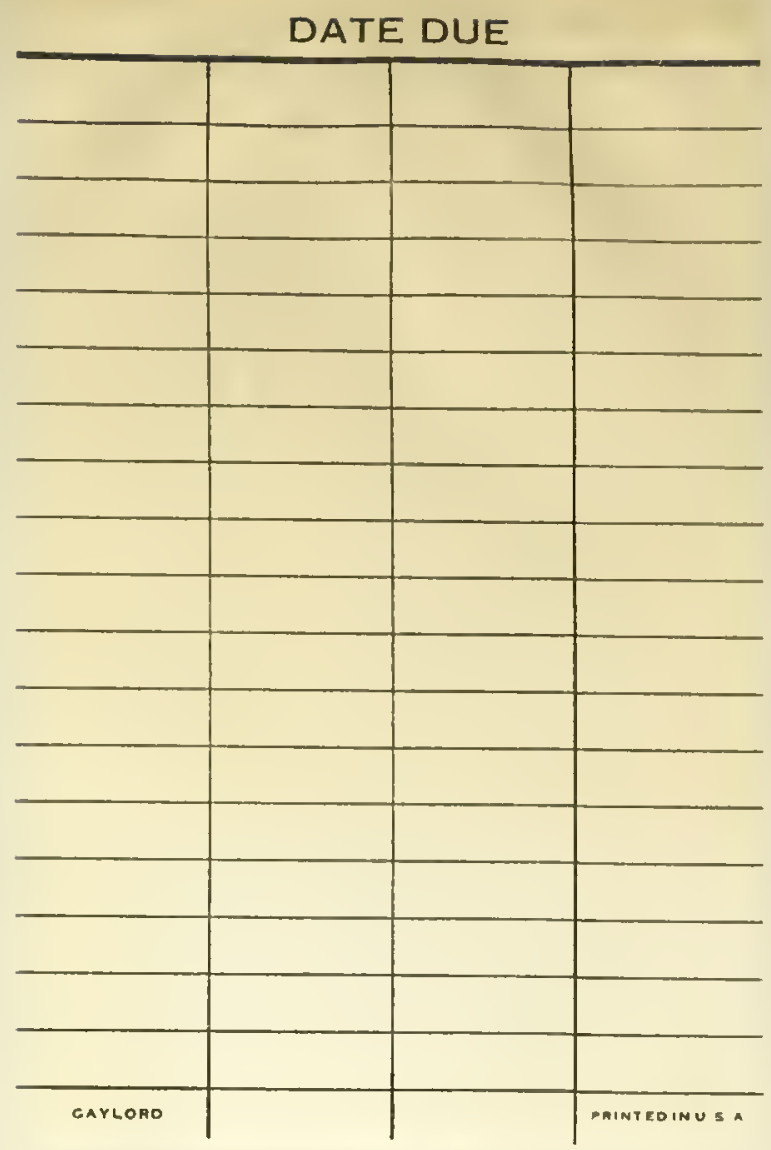

QL 595. W86 86 University Library

Coleoptera maderensla.

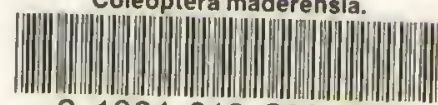

3 1924018332985 



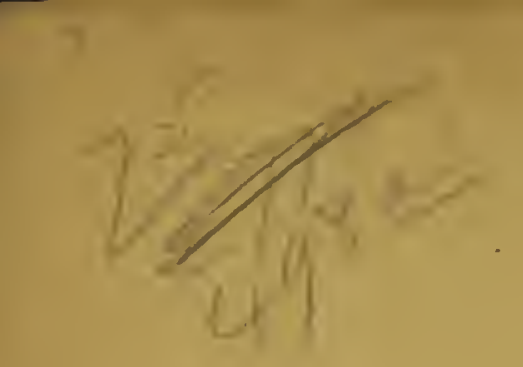




\section{COLEOPTERA MADERENSIA.}

BY

T. VERNON WOLLASTON, M.A., F.L.S.

L O N D O N :

PRINTED BY RICHARD TAYLOR AND WILLIAM FRANCIS,

RED LION COURT, FLEET STREET. 


$$
\begin{aligned}
& 21595- \\
& W 86 .
\end{aligned}
$$

$$
\begin{aligned}
& \text { QL } \\
& 595 \\
& \omega 86
\end{aligned}
$$

(a) 39842 


\section{N 'T R 0 D U C C T I 0 N.}

WIIEN we review the great questions arising out of the gcographieal distribution of animals and plants, there ean be no doubt whatsocrer that the elose investigation of any given area, howerer minute, must contribute materially, provided its position be a significant one, to lighten the labours of those more eomprehensive naturalists who are able to wield, with a master's hand, the seanty data gleaned by the humbler worker's in the scienee to a practieal aceount. And, sinec it las been said that whatsoever falls within the sphere of knowledge is attaehed to a radius and tends towards the eentre, there is reason to hope that no amount of truth, once fairly arrived at, will be eventually lost; but that it will sooner or latcr find its way into the eentral mass, to be employed, whensoever elanee may require it, for the general good. Hence it is that we are eneouraged, in crery braneh of observation, to register what we see; and to feel that the most trivial faets, if faithfully recorded, may become the basis from whence the soundest theories may arisc,- - suel theories forsooth as have already arisen from the contemplation of eireumstances apparently beneath our notice, and whieh have grown up, step by step, into trees of gigantic dimensions, to embrace at last large prineiples within their shade.

Suelı being the case, I have ventured to hope that the examination of islands even so small as those now under discussion may not have been altogether without profit. The intermcdiate situation of Madeira, whieh, whilst pertaining artificially to Europe, has nevertheless mueh in common with the north of Africa (from whieh in distance it is the less remote), imparts to it an interest, the importanee of whieh the student of Zoological geography eannot fail at onee to recognise: and, if we scan the results arrived at in the following pages, we shall perceive that there is positive ground for the belief that its Coleopterous fauna is, in a large measure, of a very isolated type. Althongh partaking, in the main, of that partieular stamp which is usually aeknowledged as Mediterranean, yet the number of endemic speeies (and cren of genera) would seem to be so great, whilst the new modifications which have becn brought to light are so extremely characteristic, 
and adjusted to the peculiar nature of the country in which they are placed, that we cannot resist the eonclusion that, whatever may have been the extent or condition of that ancient continent of which these several Atlantic elusters are the sure witnesses, that portion of it at any rate which the Madciras may be supposed to represent was not only singularly rich in ereations adapted specially to itself, but also that the various forms must have migrated but very slightly ere the land of passage was destroyed,-secing that many of them had apparently not even reached those points of its area which are now the detached portions of the actual group. That this is really a fact, we may appeat, inter alia, to such inscets as the Tarphii (only a single one of which, out of 15, occurs beyond Madeira proper), to Argutor and Treehus (of the same island), to Aealles (of which 12 members, out of 13, belong to the central mass), to the aberrant Atlantides and the Anemophili (almost exclusively Porto Santan), or to Deuealion (which reigns supreme. on the nearly inaceessible heights of the two southern Dezertas).

Although it is of eourse possible that some few out of the 270 species, and even of the 41 genera, which I have treated as novelties, may have been already made known, yet I belicre it will be found, on inspection, that such instances are rare; whilst eoneerning the elaims of the majority of them, being apparently of an endemic nature, there eannot be the slightest doubt. In addition to these 270 species, there are 11 which had been previously characterized as Madeiran; thus raising the entire number to 281 , - which, out of 482 , it must be admitted is a large proportion to possess even the ehanee of being peculiar to these islands. The genera of the present volume amount, in all, to 213: one of these (Cossyphodes) had been lately described as Madeiran; and 9 at least (namely Calobius, Daetylosternum, Xenostrongylus, Metophthalmus, Mieroehondrus, Peeteropus, Deuealion, Arthrolips and Maerostellus), out of the 41 which are indicated as new, I have reason to suspect have exponents elsewhere,-which reduces the modifications which may, or may not, be cndemic (but the larger portion of which probably are) to 34. Amongst these 34, perhaps the most remarkable are Zargus, Cossyphodes, Europs, Aphanarthrum, Leiparthrum, Eehinosoma, Xenorehestes, Glocosoma, and Ellipsodes.

It will be seen, on a referenee to the Systematie Catalogne of this work, that the total absence of numerous genera (and even of whole families) which are looked upon as all but universal, eonstitutes one of the most striking features in our entomological fauna. Thus, ineredible though it may seem, not so much as a solitary witness of the Cicindelide, Buprestide or Psclaphide has hitherto been brought to light; whilst the great gencra Carabus*, Nebria*, Silpha, Neero-

\footnotetext{
* In Dejean's Catalogue there is a Carabus registered as Madeiran, under the name of C. interruptus; and a Nebria under that of $N$. dilatata: but, as no vestige of either one genus or the other has come bencath my notice, and since they lave totally escaped the researches of the Rev. R. T. Lowe for a period of twenty-six years, as also of the late Dr. Tfeinecken and of every other naturalist (so far as I am aware) subsequently; I have not the slightest hesitation in pronouncing Dejean's insects (whatsocver they werc)
} 
phorns, Cetonia, Telephorus, Tentyria, Pimelia, Acis, Asida and Otiorhynelus are altogether wanting. 'The vast race of the Thalerophagous Lamellicorns (vid. p. 235), as also the immense department of the Elateride (vid. p. 239), are represented apparently by but a single form,-as are also the Silphide, Telephoride, Tentyriade, and the Gedemeride.

Of the 13 primary sections into which I have distributed the cntire Coleoptera, the Rhyncophora contains the largest amount of species, and the Eucerata the smallest. Arranged numerically, they are as follows: Rhyneophora (104), Necrophaga (80), Geodephaga (63), Braehelytra (74), Priocerata (35), Atraehelia (29), Cordylocerata (22), Phytophaga (21), Psendotrimera (17), Phillydirida (13), Traehelia (11), Hydradephaga (7), Eneerata (6). Now there is an anomaly in these proportions, which it is not casy, at first sight, to account for,-namely, that, whilst Madeira is essentially a land of wood and streams, the Longicorns and Water-beetles should be the least shadowed forth of the whole. As regards the latter of these, liowever, the deficiency is not difficult to understand,- the rapid nature of the rivers, which are liable to sudden inundations from the mountains, and to deposit their contents in positions distant from their banks, or to pour in ceascless torrents orer the perpendicular faces of the rocks, bcing anything but favourable to inscet life.

Of the 56 families which enter our lists, the Curculionide, Staphylinide and Carabide (as might be expected) take the lead,- - the first numbering 80 species, the sccond 73, and the third 63. The next, in point of extent, is the Colydiade, -which contains 19. The Galerucide has 13; the Lathridiade and Coccinellide 12 ; the Aphodiade 10; the Melyride 7; the Dytiscida, Histeride and Cerambieide 6 ; the Chrysomelida 4, and the Seydmenide 1 .

Of the genera with which we have here to do, Tarphius and Homalota (each of which have 15 representatives) rank first. Then comes Atlantis (which has 11); Aealles (13); Ptinus (10); Treehus and Helops (9); Bembidium and Lremophlous (8); Canlotrupis, Apion and Philonthus (7); Dromins, Corticaria, Aphodius, Longitarsus and Scymnus (6); Lixus, Sitona, Psylliodes, Coccinella and Oxytelus (5), Sc.

In glancing over our catalogue, we shall be struck, apart from the dearth in the Hydradephaga and Eucerata (already commented upon), by the great scarcity of the flower-infesting tribes,-which, in a country like Madeira, where vegetation is redundant, is not a little extraordinary. 'Tluus, to take the various families, in succession, which may be considered as par excellence falling under that denomi-

to have been ineorreetly referred (as was also, I imagine, his Melanerus Amaroides) to the islands of onr present group. They may possibly have been Canurian, or (which is more likely still) from the $\Lambda$ zores ; but until further evidenee than that of a mere Catalogne (formed in another country, and subjected to all the chanees of uneertain information) be supplied, I confess I shall not be inelined to regard them as
otherwise than apocryphal. 
nation, we find that the Phalacricla are attested by 4 Olibri; the entire Thalcrophagous Lamellicorns by a single Chasmatopterus; the Telephoride by an insignificant Malthodes; the Melyrida (which is the best indicated of the whole) by 7 spccies (contained in 5 different genera); the Clerida by an Opilus and a Neerobia (the last of which is unquestionably naturalized); the MFordellide by a solitary Anaspis; the Edemeride by a Stenaxis; and the Crioeeride by a Lema and a Crioeeris (of which the latter, if not the former also, has been imported from Europc).

Two of the principal features obscrvable throughout the Colcoptera of these islands, are the general obscureness of colouring (gay tints being exceedingly rare) and the apterous tendency. As regards the second of these, so strongly is it cxpressed, that, out of the 482 species hitherto detected, 178 arc either altogether apterous, or else have their wings so imperfectly developed that they may be practically considered as such. About 86 moleover (out of the 4.82) may, I imagine, have been accidentally introduced from other countries; and, as these belong well nigh exclusircly to the winged forms, the winged species which are in all probability truly indigenous arc diminished to 218, - - thus exceeding by only 4.0 those which are either apterous or nearly so. Numerous genera indeed (as Turus, Loricera, Calathus, Olisthopus, Argutor, Trechus, IIydrobius. \&c.) which are commonly winged are here almost invariably apterous: whilst of the converse (i.e. of insects which have their wings ample, although in other countries they are usually obsolete) there is, I believe, but a single instance,-namely Pristomyehus (concerning whicl, vide p. 218). As a corollary arising out of this peculiarity, we should a priori be led to anticipate that a large section of the Madeiran Colcoptera would be of a very loeal eharacter,-since, wherc the means of self-dispersion are reduced below the ordinary standard, a widely-acquired lange is of course next to impossible. And such, on investigation, we find to be the case,-as a glance, in fact, at the Topographieal Tables will abundantly convince.

Respecting the proportions which the sevcral islands bear to each other, in the number of species observed upon them, the great difficulties attending cren a temporary sojourn out of Madcira proper should be borne in mind, as serving to explain in some measure the impediments which surround us in arriving at any positive data on the subject. Independently however of this, the immense superficies of the central mass as contrasted with the satellites of the group,-containing as it does about ten times the area of Porto Santo (which last is, in its turn, gigantic when compared with the barren rocks of the Dezertas), and not only abounding in wood and water, but rising to nearly four times the leight,-must naturally give it an enormous preponderance in the fauna of the entire region. Still, having (at three different periods of the year) resided for more tlian a month in Porto Santo, for the sole purpose of research, and having twice encamped for at weck (in the winter and summer) on the Dezerta Grande, as well as on the Illieo Clı̃o, I belicve that I am at any rate in a position to give some sort of all opinion 
on this intricate question : and to any person who has a practical knowledge of the localities themselves, I think that the following numbers (unequal as they are) will not appear to be inconsistent with the opposite dimensions and aspects of the various portions of the cluster to which they respectively refer. Thus, in Madeira proper I have (up to the present period) asecrtained 432 species to have oceurred, in Porto Santo 111, on the Dezerta Grande 57, on the Northern Dezerta (or Ilheo Chão) 15, and on the Sonthern Dezerta (or Ilheo Bugio) 4. Or, if we choose to regard the Dezertas as onc, the group will separate itself into three natural divisions; and we shall have for Madeira proper 432, for the Dezertas 61, and for Porto Santo 111. Of the 61. species which I have found on the Dezertas, 44 have been detected in Madeira and 29 in Porto Santo. 'The species which (so far as I have becn able to ascertain) are peeuliur to Madeira proper are 340, to Porto Santo 32, to the Dezerta Grande 6, to the Ilheo Chão 3, and to the Ilheo Bugio 0.

The only insects of the existence of which I have been enabled to satisfy myself for certain on every island are the Scarites abbreviatus and the Laparoeerus morio ; nevertheless I an all but convineed that the Calathus eomplanatus, Harpalus vividus and the IIadrus einerascens (if we consider the II. illotus as its Porto Santan analogue) are equally universal: whilst, at the same time, they may he regarded, in conjunetion with the Tarus lineatus, Dromius obseuroguttatus, Olisthopus Maderensis, Omias ventrosus, Melops Pluto and eonferlus, and the Anthieus tristis, as amongst the species which are the most abundant individually of all with which we are concerned.

Taking a cursory view of the Colcoptera here described, the fauna may perhaps be pronounced as having a greater affinity with that of Sicily than of any other country which has been hitherto properly investigated. Apart from the large number of our gencra (and even species) which are diffused over more or less of the entire Mediterranean basin, this is especially evineed in some of the most characteristic forms, - -such as Apotomus, Xenostrongylus, Tarphius, Cholovoeera, Holoparameeus, Berginus, Litargus, Thorictus and Boromorphus. There is moreover, strange though it may appear to be, some slight (though decided) collective assimilation with what we observe in the south-western extremity of our own country and of Ireland,-nearly all the speeies which are common to Madeira and the British Isles being found in those particular regions; whilst one point of coincidenee at any rate, and of a very remarkable nature, has been fully discussed (vid. p. 320) under Mesites. Whether or not this partial parallelism may be employed to further Professor E. Forlhes's theory of the quondam approximation, by means of a continuous land, of the Kerry and Gallician hills, and of a huge miocene continent extending beyond the Azores, and including all these Atlantic clusters within its embrace, I will not venture to suggest: nevertheless it is impossible to deny that, so far as the Madeiras betoken, everything would go to favour this grand and comprehensire idea. Partaking in the main of a Mediterranean fauna, the northern tendency of which is in the evident rlirection of the 
south-western portions of England and Ireland, and with a profusion of endemic modifications of its own (bearing witness to the engorgement of this aneient traet with centres of radiation ereated expressly for itself, whilst geology proclaims the faet that subsidences on a stupendous seale have taken place, by which means the ocean groups were constituted; we seem to traee out on every side records of the past, and to eateh the glimpses as it were of a veritable Atlantis from beneath the wares of time,-being well nigh tempted to inquire,

"And thou, fairest Islo

In the daylight's smile,

Hast thou sunk in the boiling oeean,

While beyoud thy strand

Rose a mightier land

From the wave in alternate motion?

"Are the isles that stud

The Atlantie flood

But the peaks of thy tallest mountains,

While repose below

The great waters' flow

Thy towns and thy towers and fountains?

"Hare the oeeau powers

Made their quict bowers

In thy fanes and thy dim recesses?

Or, in haunts of thine

Do the sen-maids twine

Coral wreaths for their dewy tresses?

"But we know not where,

'Neath the desert air,

To look for the pleasant places

Of the youth of 'lime,

Whose austerer prime

The haunts of his ehildhood effaecs."

Regarding the arrangement which I have adopted, I would especially adrert to the great assistance which I have derived from Mr. Westwood's admirable Introduction to the Modern Classification of Insects, - a work the merit of whieh it is diffieult to overrate, and far surpassing every other in our own country (if not elsewhere also), in a systematie point of view, for the sound impressions whieh it conveys, and for the masterly manner in which the subject has been treated as a whole. It is a comparatively easy task to single out any one family or department, and to propound new doetrines on the eolloeation, inter se, of the various fragments which unite in eomposing it; but to weigh the problem in extenso, to balanee the diffieulties of eonflieting methods from beginning to end, and to extraet 
as far as may be possible the good from all (rejecting both what is superfluous and bad), is indeed a Gordian knot requiring a Solon to untie. And, whilst numcrous portions have been subsequently taken in hand by others, and have here and there been modified (for better or worse), the general plan which $\mathrm{Mr}$. Westwood has seleeted does still seem to offer (when contemplated in the mass) the fewest objeetions, so far as I am able to judge, of any which has been hitherto proposed. I would mention this, not because I liave altogether followed in his wake,-having departed from it in many (perliaps too many) instanees, - but simply by reason of the fact that, having made his volume my text-book ab initio, most of my ideas on the subject (and many even of the changes suggested) liave arisen from a study of its eontents : and, although I have not ehosen to eonsider myself as bound implieitly to any partieular author, yet I think it due to $\mathrm{Mr}$. Westrood to affirm that my method of arrangement has been in a very large measure moulded out of his.

The 13 primary seetions which I liave made use of are those adopted by $\mathrm{Mr}$. Westrood; nevertheless I have both transposed and inverted them, aceording as I have deemed it desirable (or where newly-discovered links rendered it neeessary) to bring ecrtain groups, formerly far asunder, into juxtaposition. Such has been the ease with the Cissida and Tomici, - a proceeding which, on account of the close affinity of the latter with the Cossonides, rendered the inversion of the Rhyncophora absolutely necessary. Then, the remoral of the Brachelytra (from the Pentamerous departments) to the end, - a step which, after much reflection, I have thought it advantageous, even on its own aceount, to take,- -has had the happy effeet of bringing Anihrenus (of the Dermestide) into direet eontact with the Byrhi, with which it has so mueh in eommon; whilst I have ventured to employ the Scydmanide (although not aetually Braehelytrous) to effect a passage from Anthicus to the Pselaphi (which apparently however have no exponent in the Madeira Islands), and from thenee (through Falagria) into the Staphylinidce. The Trogositide I have preferred to treat as a distinet family, and (for reasons stated at page 154) as more akin to the Cucujidle than to the Nitidulide,-with which it is now usually associated. The location of the Anisotomidce may perhaps require some apology; and I may add that I am not prepared to defend the situation which I lave assigned to it as of necessity the most natural one. I do, rather, in faet regard it as in reality Neerophagous, and would not willingly disturb the position (near to the Silphide) which it is generally supposed to occupy : still, the diffieulty has been felt (vid. p. 484) of diseonneeting it from the Clypeastres; and since these latter are almost universally acknowledged as inseparable from the Pscudotrimerc (an hypothesis however which I am by no means inelined to aecept as capable of positive demonstration, though I have tacitly endorsed it in the present volume), I have to a eertain degree been eoereed, contrary to my inclinations, in regulating its site.

It may perhaps be objected that I have sometimes been orer-minute in de- 
scribing my loealities, and in reeording the preeise eireumstanees under whieh many of the speeies were observed. And indeed, had I employed myself in writing for the seientifie world only, far removed from the seene of action, there would have been considerable foree in the aceusation,-for it ean clearly matter but little to the universal eolleetor to know even what island his specimens are peeuliar to (and, therefore, $\ddot{a}$ fortiori, the exact spot $i n$ that island), so long as he be fully convineed that they have come from our present Group. But let it be remembered that one of my principal designs in the following pages has been, not only to afford a complete eatalogue, to the general naturalist, of Madeiran Coleoptera, but also to put into the hands of the sojourner there for a short period (of which there are several hundreds every winter from England alone, independently of those from other countries) a full and intelligible aceount of the aetual stations in which he will probably be able to procure the several inseets required. By this means, indeed, I am emboldened to hope that my researehes may be turned to some practical aecount for the amusement of that unfortunate elass of wanderers whose lot it is to submit, year after year, to an eight months' exile in Funchal. For, plainly, to point out one way (be it but one) in whieh even a few stray minds may find an ample field to sport in during a banishment under emergenees not the most enviable, is a boon which ought not (for the salie of a useless brevity) to be overlooked, in dealing with a subject thus voluntarily undertaken (however small it be, and imperfeetly performed) for the general good.

And to those who are resident (as oceasionally happens) for a longer season than that which is ordinarily appointed for invalids, and who have health and strength sufficient to tempt them beyond the limits within which the more eautious adventurers are permitted to roam, I would add a few words, ere I elose these desultory remarks, on the pleasures of a 'Tent-life.

It will doubtless seem an insignifieant thing, when eontemplated here, to investigate thoroughly such islands as those which we are now diseussing. But the rambler in situ, who knows the diffieulties attending even a single journey to the interior, and the almost physical impossiblity of visiting many loealities except under the most auspicious eireumstanees and at partieular times, and who has perserered in vain to reach distant roeks, and failed again and again in his efforts to obtain a landing on their inhospitable shores, he alone is in a position to understand aright the numerous obstacles whieh are likely to intereept his progress. Yet sueh impediments, when surmounted, only go to inerease the satisfaction derived from the objeet attained, and give to the explorer who has suceeded in overeoming them an additional delight.

The admirer of Nature who has passed a long winter at the mountains' base, contented merely to gaze upon the towering peaks, which, though elear and eold at night, seldom reveal themselves during the day with suffieient eonstancy (through the heavy eanopy of cloud which hangs around them) to warrant an aseent, hails with unbounded joy the advanee of spring,--knowing that the time is 
at hand when he will be able to revel at large in this $A$ tlantie paradise, in remote spots seldom visited by strangers, and at altitudes where the fieree elements of winter shall give way at last to perpetual sunshine and the fresh breezes of a ealmer sea. There is something amazingly luxurious in betaking oneself to Tentlife, after months of confinement and annoyanee (it may be entirely,-partially it must be) in the heat and noise of Funchal. We are then perhaps more than ever open to the favourable impressions of an alpine existenee;-and who ean adequately tell the eestasy of a first eneampment on these invigorating hills! To turn out, morning after morning, in the solemn stillness of aërial forests, - where not a sound is heard, save ever and anon a woodman's axe in some far-off tributary ravine, or a stray bird hymning forth its matin song to the aseending sun; to feel the eool influenee of the early dawn on the upland sward, and to mark the thin elouds of fleeey snow uniting gradually into a solid bank,-affording glimpses the while, as they join and separate, of the fair ereation stretehed out beneath; to smell the damp, eold vapour rising from the deep defiles around us, where vegetation is still rampant on primxval roeks and new generations of trees are springing up, untouched by man, from the deeaying eareases of the old ones ; to listen in the still, ealm evening air to the humming of the inseet world (the most active tenants of these elevated traets); and to mark, as the daylight wanes, the unnumbered orbs of night stealing one by one on to the wide areh of heaven, as brilliant as they were on the first evening of their birth ; - are the lofty enjoyments, all, which the intelleetual mind ean grasp in these transeendent heights.

It is needless however to pursue the pieture further, for it is impossible to do justiee to what experience alone ean enable us to appreeiate. And let not any one suppose that the varied objeets and seenes of novelty which administer to our superior feelings, and eharm the eye, in these upland solitudes are adapted only to the serutiny of a naturalist, and are either beneath the notice of, or else eannot be sufficiently entered into by the general mass,-for such is by no means the ease. $\Lambda$ single trial, we are eonvineed, will be more than enough to prove the reverse, provided the adventurer be not altogether insensible to pereeptions from without, or ineurious as to the workings of the external universe around him. This however, we need seareely add, is a sine qua non,-for it has been well said that "he who wondereth at nothing hath no eapabilities of bliss; but he that scrutinizeth trifles hath a store of pleasure to his hand: and happy and wise is the man to whose mind a trifle existeth not."

The great expense necessarily attending the publieation of a work like the present one will be a suffieient guarantee that it has been undertaken purely as a "labour of love," and with the sole aim (within its preseribed limits) of arriving at the truth. How far I have sueeeded in this is a problem which must be solved by others: meanwhile I appeal boldly to observation, in situ, as the test by whieh I would most desire to be judged,--having but little fear of the experiment, and believing that we are never in so farourable a position for deeiding on the 
relative importanee of Zoological differenees as when the looal circumstanees eonnected with them are taken into aeeount. Where I have overlooked faets, or failed in my eonelusions eoncerning them, I must erave that indulgenee whieh is never denied to the honest inquirer even in a field so small as that throughout whieh my researehes have been proseeuted,-researehes whieh I am well aware ean at the best add but an iota to our knowledge,

$$
\text { "A drop disserered from the boundless sea." }
$$




\section{F A M I L I A R U II D I A G N O S E S.}

\section{Ordo I. COLEOPTERA.}

"Ala quatuor; anticis duris coriaceis, posticas membranosas (ante apiccm transversc replicatas) obtegcntibus.

$O s$ ad manducationem factum.

Metumorphosis completa." (Van der Hoeven.)

Scctio I. GEODEPHAGA ........ $\left\{\begin{array}{l}\text { Mandibula longx, exsertæ, ad apicem acutæ. } \\ \text { Maxillarum lobus externus articulatus, palpiformis; internus } \\ \text { ungue fixo terminatus. } \\ \text { Antennæe filiformes; } 11 \text {-articulatæ. } \\ \text { Pedes terrestrii (sæpius valde cursorii) ; tibiis bicalcaratis. } \\ \text { Tarsi } 5 \text {-articulati. }\end{array}\right.$

Fam. 1. Caramida ........... $\left\{\begin{array}{l}\text { MIandibula haud rel leciter (rarius ralde) dilatatæ. } \\ \text { Ligula porrceta, sapius cornea; paraglossis aucta. } \\ \text { Habitant sub lapidibus foliisque arborum dejectis, humi latitantes; ple. } \\ \text { rumque valde rapaces. }\end{array}\right.$

Subf. 1. Brachinimes.

1. Tarus (2).

2. Dromius (6).

3. Scariles (2).

4. Aputomus (1).
Elytra apice truncata (pygidium vix obtegentia), sxpius depressa. Prothorax plus minusve cordatus.

Tibice anticae intus cmarginatio.

Tarsi antici maris leviter dilatati, subtus paree squamuloso-papillosi.

\section{Subf. 2. Scaritides.} Elytra sapius clongata, subcylindrica. Mandibula plerumque valde
dentatx.

Prothorax postice contractus. Mesothorax elongatus, angustus.

Antenne articulo primo sxpius valde elougato.

Tibia antica intus emarginato, plcrumquc palmata.

Tarsi in utroque scxu simplices (rarius in mare dilatati). 
5. Calosoma (1).

6. Noliophilus (1).
7. Loricera (1).

8. Eurygnathus (I)

9. Zargus (3).
10. Pristonychus (1)
11. Calalhus (3).
12. Anchomenus (2)
13. Olisthopus (3)
14. Argutor (4).
15. Omasers (2)
16. Amara (2).

\author{
17. Anisodaclyhs (1). \\ 18. IIarpalus (1) \\ 19. Ophonus (1). \\ 20. Stenolophus (2). \\ 21. Bradycellus (2). \\ 22. Trechus (9) \\ 23. Thalassophilus (1).
}

24. Bembidium (8).

Subf. 3. Carabides.

Palpi articulo ultimo sxpius magno, truneato, subsecuriformi. Tibice omnes integræ (nee antiero emarginatæ).

Tarsi antici maris valde dilatuti.

\section{Subf. 4. Harpatides.}

Elytra apice rotundata (pygidium plerumquc obtegentia).

Tibice antice intus emarginatse.

Tarsi maris, modo antici modo anteriores dilatati.

Div. 1. CHLXNIIDEA.

Tarsi antici maris art. $2^{\text {bus }}$ vel $3^{\text {bus }}$ dilatatis (rotundatis rel quadratis), subtus dense spongiosis.

Unguiculi simplices. Pedes plerumque longiores.

Mentum vel dente medio instructum, vel cdentatum.

\section{Div. 2. Pterostichidea.}

Tarsi antici maris art. $2^{\text {bus }} \mathrm{vel} 3^{\text {bus }}$ dilatatis (cordatis vel triangularibus), subtus biscriatim setosis.

Unguiculi sæpius serrati.

Mentum dente medio (plerumque bifido) instruetum, rarius edentatum.

Div. 3. Harpalidea.

Tarsi anteriores maris art. $3^{\text {bus }} \mathrm{rcl} 4$ dilatatis, subtus plerunque biseriation setosis.

Unguiculi sæpius simplices.

ILentum dente medio (plerumque integro) instructum, rarius edentatum.

\section{Subf. 5. Bembidiades.}

Palpi articulo ultimo minutissimo, subulato.

Mentum dente medio iutegro instructum.

Tibia antice intus emarginate.

Tarsi antici maris art. $2^{\text {bas }}$ (sed præsertim $1^{\circ}$ ) dilatatis.

Sectio II. HYDRADEPHAGA... $\left\{\begin{array}{l}\text { Mandibule breves, ferc labro opertæ, ad apicem latiusculæ. } \\ \text { Maxillarum lobus externus articulatus, palpiformis (rarius ex- } \\ \text { articulatus, rariss. obsoletus). } \\ \text { Antenna filiformes (rarius subfusiformcs); } 11 \text {-articulatæ. } \\ \text { Pedes natatorii (antici interdum subambulatorii). } \\ \text { Tarsi 5-articulati (anteriores rarius art. } 4^{\circ} \text { obsolcto). }\end{array}\right.$

Maxillarum lobus externus palpiformis, biarticulatus.

Antenne longiusculæ, filiformes. Oculi duo.

Fan. 2. DrTiscrde:

Pedes natatorii (postici ad notum horizontalem solum facti).

25. Colymbeles (1).

26. Agabus (3).

27. Hydroporus (2).

Tarsi 5-articulati (anteriores rarius art. $4^{\circ}$ obsoleto).

Habitant in aquis.

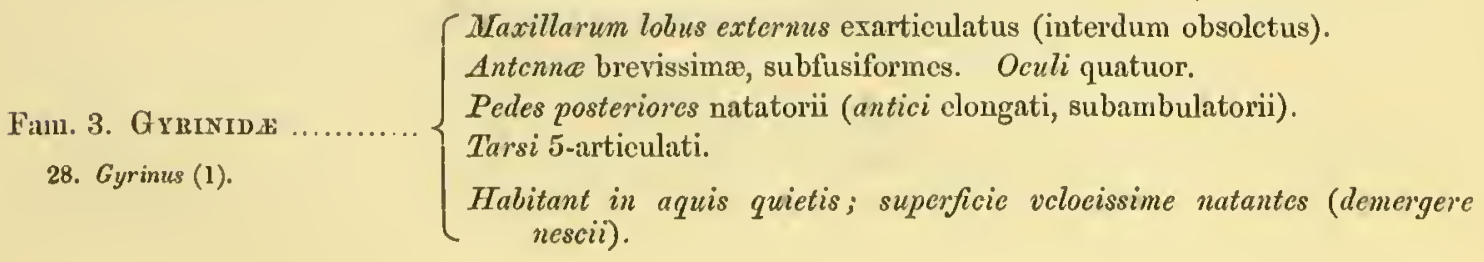




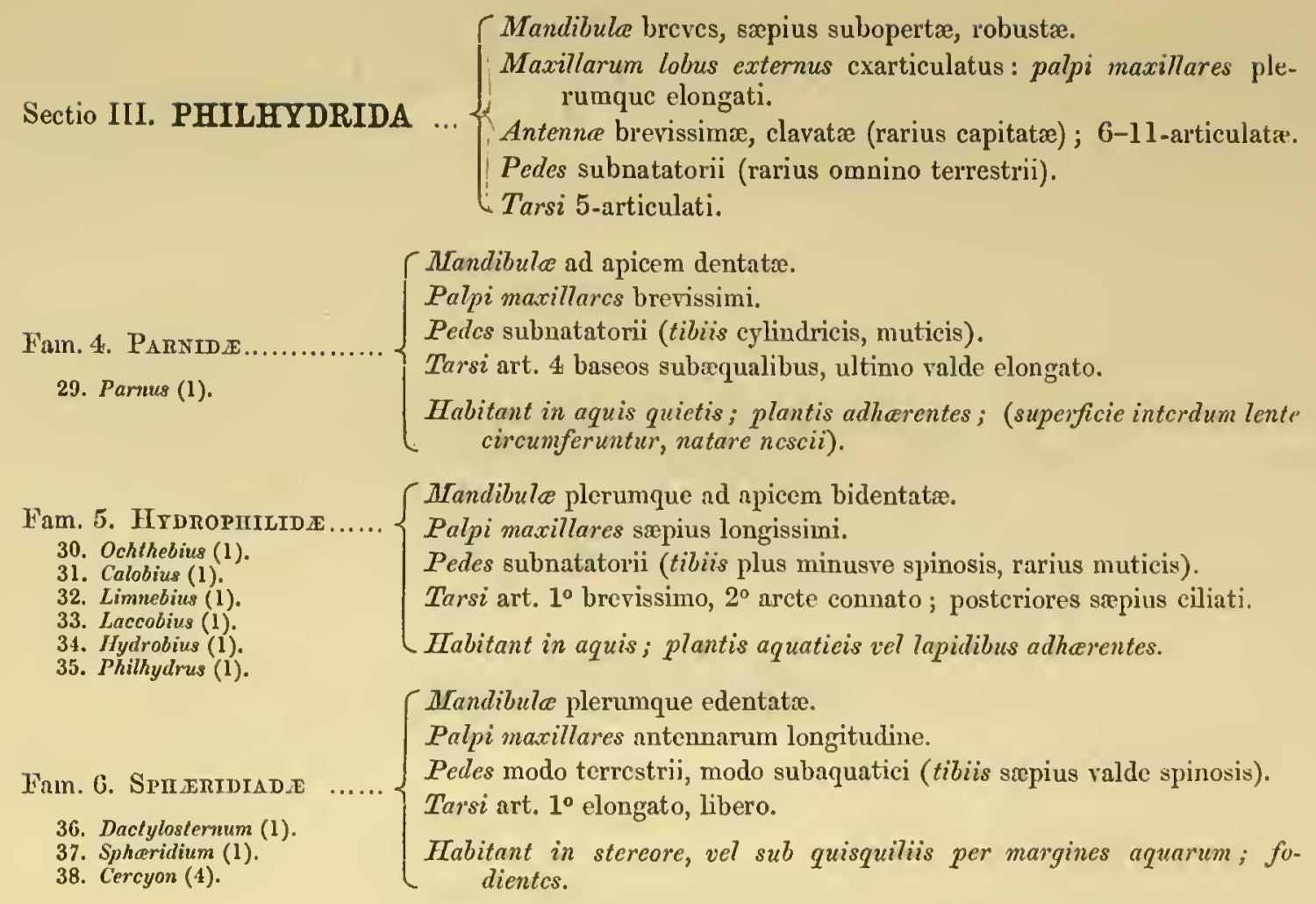

Mandibula brcves, sæpius subopertæ, robustæ.

fornus externus cxarticulatus: palpi maxillares ple-

Pedes subnatatorii (rarius omnino terrestrii)

Tarsi 5-articulati.

Habitant in aquis quietis ; plantis adharentes; (superficie intcrdum lente circumferuntur, natare ncscii).

Palpi maxillares sæpius longissimi.

Pedes subnatatorii (tibiis plus minusve spinosis, rarius muticis)

Tarsi art. $1^{\circ}$ brevissimo, $2^{\circ}$ arcte connato ; posteriores sæpius ciliati

Habitant in aquis; plantis aquatieis vel lapidibus adherentes.

Iandibula plerumque edentatx.

Pal maxillares antennarum lon ritudue.

Tarsi art. $1^{\circ}$ elongato, libero.

dientcs.

Fum. 7. Silpindde 39. Catops (1).
Fam. 8. Prillade 40. Acratrichis (3). 41. Plenidium (1).

Fain. 9. Pinatancride 42. Olibrus (4).
Maxillarum lobus externus exarticulatus (rarius obsolctus). Antenne clavatie vel capitatx.

Pedes terrestrii (interdum subcontractiles).

Tarsi sæpius 5 -articulati.

Maxilla bilobæ.

Antcnne 11-art., apicem rersus sensim incrassatæ, rel claratı (clavâ 4-5-art.).

Abdomen e segmentis ventralibus 6 compositum.

Pedes sxpius subgraciles, leviter elongati.

Tarsi 5-articulati.

ILabitant in cadaveribus putrescentibus, quisquiliis, vel sub lapidibus; vorantcs.

Maxilla bilobs: palpi maxi7lares art. ultimo minutissimo, aciculari.

Antenne 11-art., capillares, șubclavatic (clavâ 3-art.).

Abdomen e segmentis ventralibus 5-7 compositum.

Corpus minutissimum; atis lanceolatis, amplissimis, longissime cilintis.

Pedcs gracillimi.

Tarsi 3-articulati.

IIabitant in quisquiliis, sub foliis arborum dejectis, vel in terrâ lumida, velocissime eursitantes.

Maxilla bilobr.

Antenne 11-art., clavatæ (clavâ 3-art.).

Abdomen e segmentis ventralibus 5 compositum.

Pedcs graciles.

Tarsi 5-articulati (art. $4^{\text {to }}$ minutissimo).

IIabitant inter flores vel in graminosis; veloeissime eursitantes. 


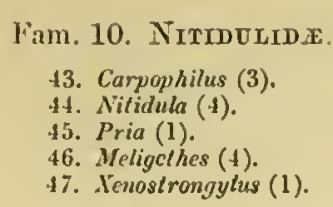

Fam. 11. Colydidde........ 18. Tarphius (15). 49. Cossyphodes (1)

50. Plceosoma (1).

51. Europs (1).

52. Lyclus (1).

Fam. 12. Thogositine 53. Trogosita (2).
(Maxilla lobo singulo instructro (rarius bilobre).

Antenne 11-art., capitatre (capitulo sæpius 3-art.).

Abdomen e segmentis ventralibus 5 compositum.

Pedes robusti, breviuscnli, subcontractiles.

Tarsi 5-articulati (art. $4^{\text {to }}$ minutissimo).

Habitant in floribus, sub eortice arborne, inter fungos, vel etiam in ossibus (eartilaginem arrodentes).

MLaxilla bilobs.

Antenne 10-11-art., clavatie vel capitatio (clavâ sæpius 2-vel 3-art.). .

Abdomen c seguncntis rentralibus 5 compositum.

Pedes parum graciles, sxpe subcontractiles.

Tarsi 4-articulati (rarius subconici).

Ilabitant sub truncis corticcve arborum marcido, inter lichenes; vel in tenebris latentes.

Maxillac lobo singulo instructx (interno obsolcto).

Antenne 11-art., filiformes rel subclavatæ.

Corpus plus ninusve clongatum, depressum.

Pedes sat robusti, prosertim antiei.

Tarsi srepius 5-articulati (art. $1^{\circ}$ minimo).

IIabitant eirea granaria et domos; inter oras diversas per commcrcium sape translate.

(Maxilla bilobæ (lobo interno sæpius minutissimo).

Antennce 11-art, filiformes rel subclavatx.

Corpus plus minusve clongatum, plcrumque valde depressum.

Pedes parum graciles, antici sæepius robustiores.

Fan. 13. CucuJide

54. Ciryplamorpha (1).

55. Leemophleus (8).

56. Silvanus (3).

Tarsi sæpius 5-art., in maribus intcrdum heteromeri; (art. $1^{\circ}$ plcrumquc minimo).

Habitant sub cortice arborum, in granariis vel circa domos; commereium interdum sequentes.

Mraxilla bilobæ.

Antenne 11-art., clavatæ.

Corpus plus minusve oblongo-oratum, convexiusculum.

Pedes sxpius parum graciles.

Tarsi 5 -articulati, in maribus interdum heteromcri.

Habitant in fungis, quisquiliis, val etiam in domibus; interdum semina destruentes.

9. Diphylus (1).

60. Ephislcmus (1).

Jam. 15. LatinidIADE .....
61. Cholnocera (1).
62. Hotoparamecus (1).
63. Corlicaria (6).
61. Lalhridius (3).
65. Helophlhatinus (1).

(NTaxilla bilobæ (lobo interno sæpius obsolcto).

Antennce 8-11-art., clavatæ.

Corpus minutum, plus minusre oblongo-ovatum, convexum vel depressum. Pedes subgraciles.

Tarsi sæpius 3-articulati (antici interdum 4-art.).

ILabitant sub lapidibus, cortice, in locis subterraneis, vel in formicarum nidis; eurrentes.

IIaxilla bilobæ.

Antennce 11-art., clavatre.

Corpus plus minusve oblongo-ovatum, convexiusculum, pilosum, pictum.

Pedes parum graciles.

Tarsi 4-articulati (antici in maribus sxpius 3-nrt.).

66. Berginus (1).

67. Microchondives (1).

68. Typhan (1)

69. Litargus (1).

Ilabitant inter fungos, sub eortice arborum, vel in ligno antiquo; sapius agilitcr moventes. 


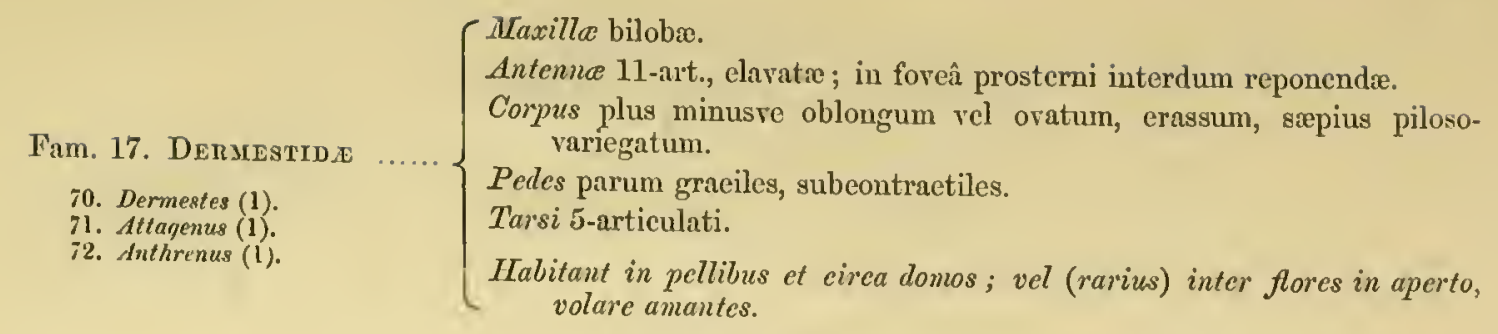
Maxillarum lobus externus exarticulatus; internus vel minutus vel obsolctus. Sectio V. CORDYLOCERATA... $\left\{\begin{array}{c}\text { Antenne breves, capitatæ, serrato-, vcl lamellato-elavatæ (sæepe } \\ \text { geniculatæ) ; 8-11-art. }\end{array}\right.$
Pedes terrestrii (sxpe omnino contractiles).
Tarsi 5-articulati.

Fam. 18. Brrmind:

73. Syncalypta (3).

Fam. 19. Histeride

74. Hister (1).

75. Paromalus (2).

76. Saprinus (3).

Fam. 20. Thontctid

77. Tharictus (1).
Maxilla bilobæ. (Mandibula vix exsertæ.)

Antenna 11-art., elaratre, breses; in foveâ prostcrui reponendx.

Corpus ovatum, crassum, serieeo-pilosum; prosterno antiec producto; alis rarius obsoletis.

$\{$ Pcles robusti, eontractiles; (femoribus tibiisque longitudinaliter exeavatis).

Tarsi 5-artieulati, ad tibias reponendi.

Ilabitant in graminosis, sub lapidilus, vel in arenosis ; propter hunum lente repentes.

Maxille bilobæ. (Mandibula sæpius magnæ, exsertæ.)

Antenne 11-art., eapitats, breres, genieulatæ; scapo longissimo, in fovêt sub margine eapitis reponendo.

Corpus rotundato-quadratum, durum, glabcrrimum; prosterno antice sæpius producto; clytris truncatis.

Pedes robusti, contractiles; (tiliis plus minusre longitudinaliter excavatis et dentatis).

Tarsi 5-articulati, ad tibias sæpius reponendi.

Habitant in cadaveribus et quisquiliis, vel etiam sub lapidibus; lente repentes.

Maxilla bilobr. (Mandibula vix exscrtre)

Antenne 11-art., enpitatx, brevissimæ, robusta; ad marginem eapitis reponeudx.

Corpus obtuso-subovatum, durum, politissimum ; mesosterno brevissimo, scutello vix observando; alis obsoletis.

Pedes robustissimi, subcontractiles (tibiis setosis), ad basin ralde approximati.

Tar'si 5-artieulati, breres, subconici.

ILabitant in formiearum nidis, vel sub lapiditus; latentes.

Marilla bilobæ. (Mandibula labrumque membranaceun clypeo opertr.)

Antenna 9-art., lamellato-elaratæ, breres; in foveâ ad marginem eapitis reponenda.

Corpus plus minusre oblongum, conrexum; seutcllo distincto.

Pedes robusti, subeontractiles; (tibiis antieis tridentatis, posterioribus setosis).

Tarsi 5-articulati, ad tibias reponendi.

IIabitant in stereore, quisquiliis, vel in arenosis ; fodientes. 
Fam. 22. Troolde

81. $\operatorname{Tr} a x(1)$.

Maxilla bilobr. (Mandibula labrumque inæqualc erustaceum clypeo liaud opertre.)

Antennce 9-10-art., lamcllato-clavatx, breves; in foveâ ad marginem eapitis reponcudx.

Corpus ovatum, crassum, tuberculato-rugosum ; scutello distincto.

Pcdes parum robusti, subcontractiles; (tibiis setosis, anticis obscure dentatis).

Tarsi 5-articulati.

Habitant quisquilias in arenosis; interdum ctiam ad ossa allecte (cartilaginem arrodentes).

(Naxilla lobo singulo dcutato instrnctx. (Mandibula subopertx. Labrum crustaceum exsertum.)

Antenne 9-10-art., lamellato-elaratæe, breves; in foveâ ad marginem capitis reponendre.

Fam. 23. Graphimid

Corpus oblongum, subcourexun, pilosum; scutello distincto; elytris leviter truncatis.

82. Chasmatopterus (1).

Pedes clongati; (tibiis anticis ad apicem internum oblique truncatis, excavatis).

Tarsi 5-articulati, graciles; unguiculis sxpius dentatis vel bifidis.

L Habitant super plantas; flores foliaque devorantes.

Seetio VI. PRIOCERATA ...... $\left\{\begin{array}{c}\text { Maxillarum lobus externus exarticulatus. } \\ \text { Antennæ mediocres, filiformes, serratæ vel peetinatæ (rarius } \\ \text { clavatæ); sxpius 11-art. } \\ \text { Corpus modo durum, prostcrno produeto; modo molle, prostcrno } \\ \text { simplici. } \\ \text { Pedes terrestrii (interdum subcontractiles). } \\ \text { Tarsi plerumque 5-articulati. }\end{array}\right.$

(Maxilla bilobx. (Mandibule leriter exsertx.)

Antenna 11-art., clavatæ, breves ; in foveâ prostcrni reponenda.

Corpus ellipticun, durum ; prothorace ad angulos posticos valde produeto,

Fam. 24. Tirnoscride prosterno autice producto.

83. Trixayns (1).

Pedes graciles, contractiles.

Tarsi 5-artieulati, graciles, reecpti.

Habitant in foliis arborum, inter lichcnes, vel in arenosis; currentes.

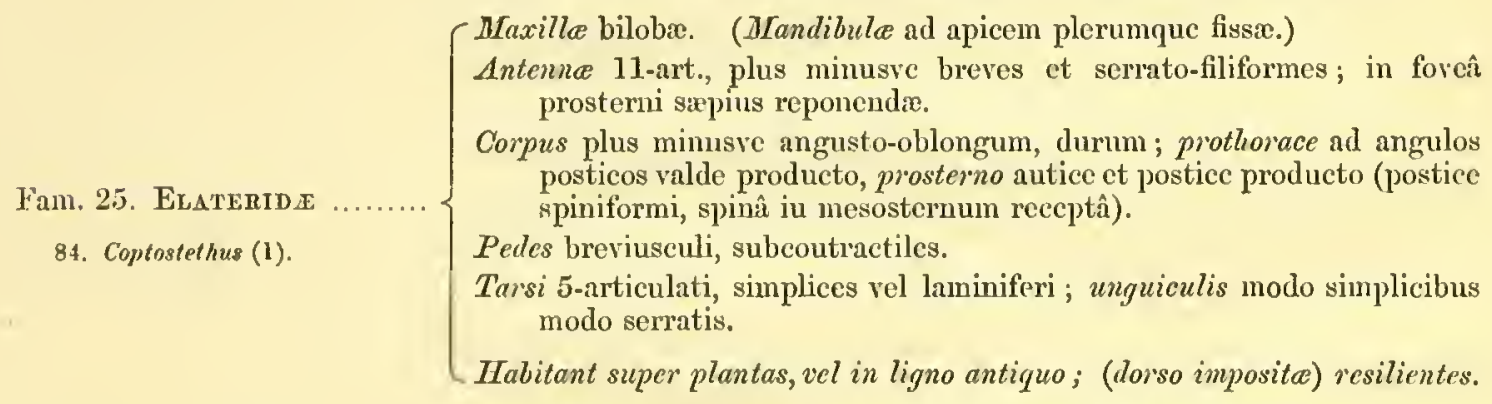

MFaxilla bilobæ. (Palpi labiales in typicis fureati, sed iu genere nostro simplices.)

Antenne 11-art., breves, filiformes vel subserrata.

Fam. 26. Стрногіd ж........

Corpus plus minusve ovatum vel hemispharicum (in typicis molle); prostcrno simplici.

85. Eucinetus (1).

Pedes sat graciles, postici interdum saltatorii.

Tarsi 5-articulati.

Habitant in paludosis, vel etiam sub cortice laxo; interdum agre saltuners. 


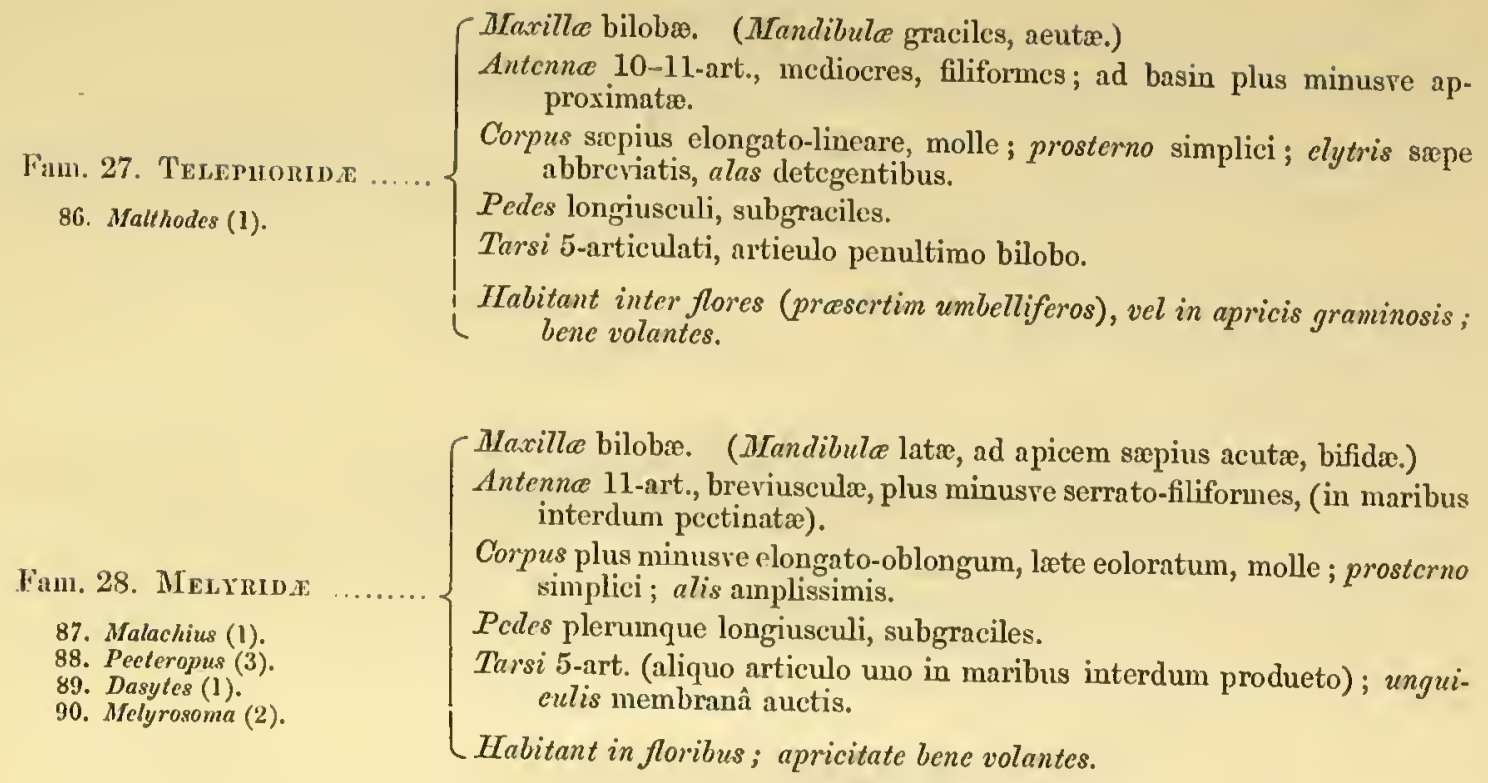

Fam. 29. Cleridx

91. Opilus (1). 92. Necrabia (1).
Maxilla bilobx. (Mandibula infra apicem sxpius unidentatæ.)

Antcnnce 11-art., breves, plus minusvc clavatæ (rarius scrrato-filiformes). Corpus clongato-subcylindrieum, pilosum, læte eoloratum, punctatum, durusculum; prosterno simplici.

Pedcs longiusculi, parum robusti, rarius subeontraetiles.

Tarsi 5-art. (modo omnes, modo aliquo pari uno pscudotetrameri), art. $3^{\circ}$ vel $4^{\circ}$ sæpius bilobo.

Habitant inter flores, in ligno antiquo; vel etiam in ossibus (cartilaginem rodentcs).

Mraxilla bilobw (lobo interno lato). (Mandibule dente plus minusve medio, obtuso instructæ.)

Antenne 11-art., breviuscula, filiformes rel subclaratæ; sæpc ad basin approximatie.

Fam. 30. PTINIDA

Corpus plus minusve orbieulato-ovatum vel oblougum, durum; prosterno simplici; capite deflexo.

Pcdes longiusculi, graciles rel robusti, plerumque subcontraetiles; (tibiis simplicibus).

94. Prinus (10)

95. Giblium (1).

96. Anobium (4).

Tarsi 5-articulati.

IIabitant inter pelles, cirea domos et in ligno antiquo; vel inter liehenes in aperto latcutes.

Mraxilla bilobse (lobo interno plcrumque minutissimo). (Mrandibula ad apiecm sæpius bidentatæ.)

Antenna 8-11-art., clavatro (clavâ laxâ 3-art.), breves, distantes, (rariss. flabellato-serrata).

Corpus subcylindricum, durum; protleorace antiee produeto, interdum rugoso, prostcrno simplici; capite deflexo.

Fan. 31. Cisside

Pcrles breviusculi, subcontraetiles; (tibiis simplicibus, vel apieem versus cxtermum dentatis).

98. Oefotemnus (1).

99. Pitinus (1).

100. Rhyzopertha (1).
Tarsi 4-, vel 5 -artieulati.

Mabitant in fungis, ligno antiquo, vel (rarius) circa domos; sapius terebrantes. 
Sectio VII. RHYNCHOPHORA.

Labrum sxpius obsoletum.

Maxilla lobo singulo lato exartieulato plerumque instruetæ (intorno obsoleto).

Antenne sxpius genieulata (art. $1^{\circ}$ elongato), eapitata vel elavatx; $y-12$-art.

Corpus plus minusve elongato-ovatum vel cylindrieum; eapite (prixsertim in maribus) rostrato.

Pedes terrestrii (rarius subcontraetiles); tibiis uneinatis vel simplieibus.

Tarsi pseudotetrameri (i. e. 5 -art., art. $3^{\circ}$ bilobo $4^{\text {tum }}$ minutiss. reeipiente), rariss. simpliees.

Fam, 32. Tomiciv

101. Tomicus (2).

102. Aphanarthrum (1).

103. Leiparthrum (4).
Fam. 33. Hruestridx

104. Phlceophthorus (1).

105. Hylurges (2)

106. Hylastes (2).
Fam. 34. Curculonda

MLaxilla lobo singulo lato setoso instruetæ (interno obsoleto). (Mandibulce latæ, obtusæ.)

Labrum obsoletum. Palpi (præsertim maxillares) erassi, eoniei. Ligula elougata.

Antenno 8-11-art., eapitatæ, breves, geniculatro (seapo longissimo), ad marginem eapitis inserta.

Corpus eylindrienu; prothorace antiee produeto, sæpius rugoso; capite deflexo, vix rostrato.

Pcdes brevissimi, robusti, subeontraetiles; (libiis sxpius eompressis, extus deutatis).

Tarsi 5- (rariss. 4-) art., simpliees (art. $4^{0}$ saltem minutissimo), ad tibias reponeudi.

Habitant in ligno, vel sub cortice arborum; valide terebrantes.

Mlaxille lobo singulo lato setoso instruetæ (interno obsoleto). (Mandibula lata, obtusa.)

Labrum obsoletum. Palpi (præsertin maxillares) erassi, eomiei. Ligula elongata.

Antenne 8-11-art., eapitatæ, brevos, genieulatæ (seapo lougissimo), ad marginem capitis insertæ.

\{ Corpus ovatum rel eylindrieum; prothoraec leviter produeto, rarius rugoso; eapite detlexo, seusin rostrato.

Pedcs breves, robusti, subeoutraetiles; (tibiis sæpius eoupressis, extus dentatis).

Tarsi pseudotetrameri, ad tibias reponendi.

Habitant in ligno, vel sub eortice arborum; valide tercbrantes.

Mlaxille lobo singulo lato setoso instruetx (interno obsoleto). (Mandibule latæ, obtusiuseulæ.)

Labrum obsoletum. Palpi crassi, coniei. Ligula elongata.

Antenne 7-12-art., elavatx rel eapitatx, genieulatx (seapo longissimn), rostro scrobieulato insertx.

Corpus plus minusve elongato-ovatum, eonvexum; eapite sæpius deflexo, (interdum valde) rostrato.

Pedes modiee elongati, rarius subeontratiles; (tibiis vel siuplieibus, vel ad apieem uneinatis).

Tarsi pseudotetrameri.

IIabitant super arbores et plantas; folia, scmina, vel etian ramos, destruentes.

Div. 1. $\left\{\begin{array}{c}\text { Rostrum eylindrieum vel filiforme, plerumque elouga- } \\ \text { tum (rarius thoraee brevius). } \\ \text { Antenno ante vel pone medium rostri (uee juxta sinum } \\ \text { oris) inserta. }\end{array}\right\}$ Mceorlynehi. 


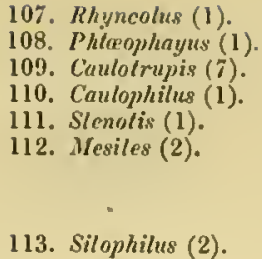

113. Silophilus (2).

114. Cionus (1)

115. Ceutorhynchus (-1) 116. Cueliodes (1) 117. Acalles (13)

118. Tychius (3) 119. Pissodes (1). 120. Lixus (5).

121. Cyphoscelis (1). 122. Laparocerus (1) 123. Allantis (14). 124. Omias (3)

125. Anemophilus (3).

126. Lichenophayus (2)

127. Scoliocerus (2).

128. Trachyphloets (1).

129. Echinosoma (1).

130. Hypera (3).

131. Cleonus (1)

132. Silona (5)
Subf. 1. Cossontdes.

Antonnce breves; funiculo 7 -art.; clarâ subsolidâ, ad apieem spongiosâ. Pedes antici ad basin distantes rel approximati.

Subf. 2. RTrNciropHorumes.

Antonno medioeres; funiouto 6- (rarius 5-) art.; clavá subsolidâ vel 2 -art.

Pedes antiei plenumque paulo longiores.

\section{Subf. 3. Cionides.}

Antenno breviuseux; funiculo 5-art. ; clava 3-, rel 4-art.

Pedes antiei ad basiu vel approximati vel distantes.

\section{Subf. 4. Criptormincmides.}

Antcnna medioeres; funiculo 7-art, ; clavd 4-art.

Rostrum inflexum, in eanaliculam peetoralem distinetan applicandum.

Pedes antici ad basin distantes.

\section{Subf. 5. Erirhinides.}

Antenno mediocres; funiculo 7 -art.; clavá 4-art.

Pedes antici ad basin approximati.

Div, 2. $\left\{\begin{array}{c}\text { Rostrum plus minusve erassum et deforme, brevius- } \\ \text { euluu. } \\ \text { Antonnc prope apieem rostri (sæpe juxta sinum oris) } \\ \text { insertie; sæpissime } 12 \text { art }{ }^{\mathrm{t}} \text {. }\end{array}\right\}$ Brachyrhynehi.

Subf. 6. CrClomines.

Canalicula antennalis subreeta, rersus medium rostri aseendens.

Rostrum breve, subhorizontale, lineare, teretiuseulum (nonnunquam apjieem versus subattenuatum).

Corpus plerumque brevius, suboratum, apterum.

Subf. 7. Brnsopsides.

Canaticula antcnnalis infra-oeularis, eurvata rel obliqua.

Rostrum breve, inflexum, in eanalieulam peetoralem plerumque applieandum.

Corpus sxpius ovatum, eonvexum, inxquale, squamosum, apterum ; seutello nullo.

Tarsi plerumque angustati, setosi.

Subf. 8. MToLytides.

Canaticula antemalis infra- (rel subinfin-) oeularis, eurvata vel obliqua.

Rostvum lougins, deflexum, subeylindrieum, paulo areuatun.

Corpus plus miuusve oblongum, squamosum et pubeseens, apterum vel alatum.

\section{Subf. 9. CLEONIDES.}

Canatieula antennalis infra-oeularis, eurvata rel obliqua.

Rostrum longiuseulum, deflexum, apiee sæpius subinerassatum.

Corpus plerumque sat magnum, squamosum et pubeseens, alatum rel apterum.

Subf. 10. Bractirderides.

Canalicula antennalis infra-oeularis, eurvata rel obliqua.

Rostrun breve (iuterdum brevissimum), sublorizontale, fere eapitis latitudine, planiuseulum.

Corpus elongato-oblougum (rarius oratum), alatum vel apterum. 
Fam. 35. ATrelabide 133. Apion (7). 134. Auletes (1)

Fam. 36. Bruchid.

135. Xenorchestes (1).

136. Bruchus (3).
MLaxilla lobo singulo lato setoso instructie (interno obsoleto). (Mandibula latæ, obtusiusculæ.)

Labrum obsolctum. Palpi crassi, conici.

Antenne 11-12-art., clavatæ vel subfiliformes, rectæ, rostro vix scrobiculato inscrtie.

Corpus sxpius ovatum, convexum; eapite subdeflexo, (sæpissino valdc) rostrato.

Pedes modice elougati; (tibiis plerumque simplicibus).

Tarsi pseudotetrameri.

Habitant super plantas et arbores; folia devorantes.

Mfaxille bilobæ. (Mandibule robusta, acutiusculæ.)

Labrum distinctum. Palpi sat elongati, filiformes.

Antenne 11-art., subfiliformes vel clavate, recte, rostro hand scrobiculato insertax.

Corpus rotundato-oratum, convexum; eapite deflcxo, levitcr rostrato, lato; elytris sxpius abbreviatis.

Pedes modice elongati; (tibiis plerumque simplicibus) : postici intcrdum validiores.

Tarsi pseudotctraneri.

Habitant super plantas, semina destruentes; inter liehenes, vel (ravius) sub eortice arborum laxo.

\section{Subf. 1. Anturibides.}

Antenne apicem versus plcrumque clavatæ, (in maibus interdum longiores).

Oeuli integri.

Pedes postici haud validiores (sed rariss, subsaltatorii).

\section{Subf. 2. Bruchides.}

Antenne filiformes, aut apicem versus leviter incrassatæ et sæpius subserratio.

Oeuli lunati (i.e. intus profunde cmarginati).

Pedes postiei plerumquc validiores.
Sectio VIII. EUCERATA

Fam. 37. Ceraybicida

137. Stromatium (1).

138. Phymatodes (1).

139. Blabinolus (1).

140. Tricheferus (1).

141. Clyties (1).

142. Deucalion (1).
Labrum exsertum (rariss, obsolctum).

Maxillarum lobus externus cxarticulatus; internus distinctus (rariss. obsolctus).

Antenne plus minusve longissima, filiformes vel sctacex (rarius serratx); sxpius 11-art.

Corpus plerumque magnum, clongatum; oculis sxpius intus emarginatis.

Pedes terrestrii, longiores; (femoribus sæpc clavatis).

Tarsi pseudotetrameri.

Maxille bilobx (lobo interno sat magno), submembranacca.

Antenne sæpius 11-art., longissima, fliformes vel scrratæ, ad marginem oculorum intcrnum insertæo.

Corpus maguum, plus minusve parallelum; eapite modo porrecto, modo deflexo.

Pedes clongati; (femoribus plus minusve clavatis).

Habitant intra lignum antiquum, sub cortice, vel infloribus; sapius bene volantes. 

Maxillarum lobus externus sæpius subarticulatus, pseudopalpi- formis.
Seetio IX. PHYTOPHAGA
Antenna breviusculæ, filiformcs vel leviter inerassatæ, plus minusve approximatæ; sæpius 11 -art.
Corpus ovale, crassum (rarius elongatum), sæpius læte coloratum et glabrum.
Pedes terrestrii ; (postici interdum saltatorii).
Tarsi pseudotetrameri.

Fam. 38. Criocentide

143. Lema (1).

144. Crioceris (1).

Fan. 39. Cassidide

145. Cassida (2).

Fam. 40. Galendoide $\ldots .$.

146. Ilaltica (2).

147. Longitarsus (6)

148. Psylliodes (5).

\section{Fam. 41. Chrsomelid}

149. Mniophilosoma (1).

150. Cryptocephalus (1).

151. Chrysomela (1).

152. Gastrophysa (1).
Maxilla bilobæ (lobis latis subæqualibus, externo land palpiformi), submembranaccæ.

Antenne 11-art., apicem versus sæpius vix incrassatre, ad basin parum distantes.

Corpus plus minusve elongato-oblongum, parallclum, pictum; abdomine amplo.

Prothorax elytris angustior, sæpius subeylindricus.

Pedes sat elongati; (femoribus posticis interdum incrassatis, dentatis ; tibiis sæpe subcurvatis).

Ilabitant in plantis, prasertim subaquatieis, vel inter flores; folia ct ramos destruentes.

(Maxilla bilobæ (labo ext angusto, reeto, subpalpiformi ; int parro), cum labio, membranacer.

Antennce 11-art., breves, apicem versus sensim inerassatie, ad basin approxinlatæ.

Corpus latum, subtus deplanatum, plus minusve rotundatum; prosterno antice leviter producto.

Prothorax et elytra ad latera valde producti; i7lo semicirculari, caput obtegente.

Pedes breves, retractiles; tarsis latiusculis (art. $3^{\circ}$ longe bilobo, $4^{\text {tum }} 5^{\text {tumque }}$ includente).

Habitant super folia plantarum, precipue in locis humidiusculis; lente repentes.

Mraxilla bilobro (lobo ext angusto, fracto, subpalpiformi; into lato, magno), membranacex.

Antenne 11- (rariss. 10-) art., longiuseulæ, subfiliformes, ad basin approximatæ.

Corpus plus minusve oratum, convexiusculum.

Prothorax et elytra basi latitudine vix aquales.

Pedes sat graeiles, longiusculi ; (femoribus posticis sxpissime inerassatis, saltatoriis).

Irabitant super folia plantarum, presertim in graminosis ; plerumque fortiter salientes.

Maxille bilobø (lobo ext sxpius subpalpiformi, incurvo), submembranacex.

Antenna 11-art., breviusculæ, filiformes vel leviter inerassatæ, ad basin distautes.

Corpus rotundato-, rel subcylindrico-oratum, convexum, erassum, sæpe splendore superbiens.

Prothorax et elytra basi latitudine xquales."

Pedes sat robusti, subretraetiles; tarsis latiuseulis.

IIabitant in foliis plantarum ; apricitate gaudentes. 
Sectio X. PSEUDOTRIMERA. $\left\{\begin{array}{c}\text { Maxillarum lobus cxternus exarticulatus; internus interdum ob- } \\ \text { soletus. } \\ \text { Antenna plus minusve brevissimæ, clavatæ (rarius subfiliformes) } \\ \text { sepius } 11 \text {-art. } \\ \text { Corpus ovalc vel hemisphæricum, glabrum aut tenuitcr pubescens. } \\ \text { Pedes terrestrii (sæpius snbcontractiles). } \\ \text { Tarsi pscudotrincri (i. c. } 4 \text {-art., art. } 2^{\circ} \text { bilobo, } 3^{\text {um }} \text { minutiss. re- } \\ \text { cipientc). }\end{array}\right.$

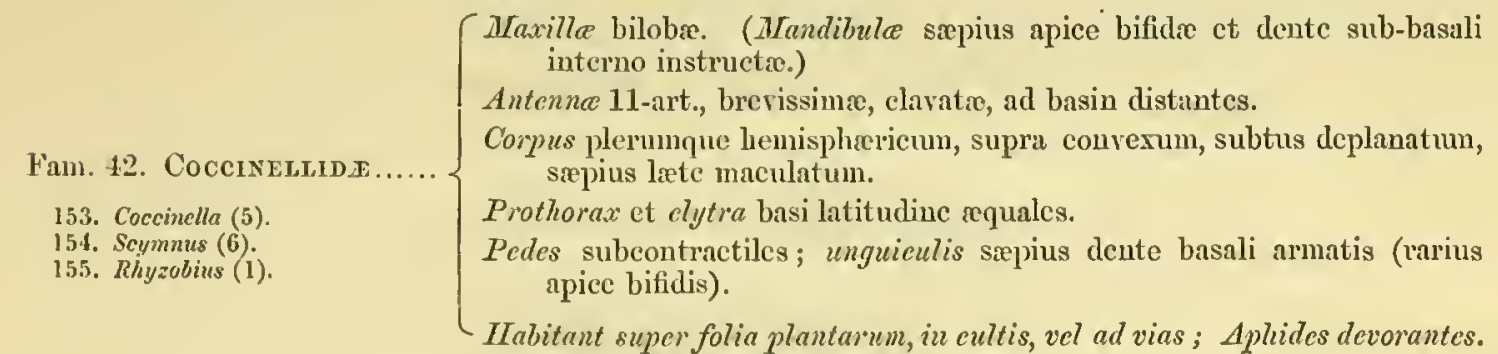

CMaxillec lobo singulo angusto, clongato, recto, apice denticulato, instructæ (interno obsolcto).

ILandibula plerumque apice denticulatio, per marginem internum interdum crenulatæ.

Antennce 9-11-art., breviusculæ, clavatæ vel subclaratæ, ad basin distantes vel subapproximatic.

Fam. 43. ContlopHide......

Corpus ovatum vel hemisphæricum, minutum, sxpius supra et subtus subconvexum; alis plerunque amplis ciliatis.

156. Clypeaster (1).

157. Arthrolips (1).

158. Sericoderus (1)

159. Corylophus (1)

160. Gloeosoma (1).

Prothorax et elytra basi latitudine æquales; illo ad latera ct antice produeto, caput obtegente.

Pedes graciles, subcontractiles; (postici valde distantes).

Tarsi 4-articulati, simplices.

Habitant inter plantas (precipue Endogenas) sub fibra stirpium, vel sub folia dejecta ; cursitantes.

Mandibula sxpius ad apicem bifidx, et in medio fisso-sinuatx.

Maxillarum lobus externus cxarticulatus : palpi max. art. ultimo sæepius securiformi.

Antcnne plcrumque breviuscule, filiformes, apice levitcr incrassatæ (rarius clavatæ); sæpius sub frontis margine inscrtæ

Sectio XI. ATRACHELIA ..... $\left\{\begin{array}{c}\text { ct ll-art. } \\ \text { Corpus durum, plerumque haud pilosum et obscure coloratum; }\end{array}\right.$ capite in cavo prothoracico usque ad oculos immcrso.

Pedes terrestrii; tibiis bicalcaratis, ct sæpius ad apicem minute spinulosis.

Tarsi hetcromeri (i. c. anterioies 5-, postici 4-art.) ; rariss. omncs 5 -, vel 4-art.

DIaxille bilobæ (rariss. Tobo singulo instructx). (MIandibula apice integra rel bifidis.)

Antennce 9-11-art., breviusculx, clavatæ (articulo clavæ secundo sxpe minuto).

Fam. 44. Arisotomids......

161. Stagonomoryha (1).

Corpus plus mimnsre orbiculato-oratum, glabrum; eapite sæpe ad pectus arcte applieando.

Protloorax et elytra valde conrexi, basi latitudine acquales.

Pedes subcontractiles; (tibiis plus minusve curvatis et spinosis).

Tarsi modo 4-, modo 5-articulati, modo leteromeri.

Habitant in unbrosis lumidis, sub truncis arborum maveidis, vel inter quisquitias; cuisitantes. 
Fam. 45. Diaperida

162. Ellipsodes (1).

163. Phaleria (1).

Fam. 46. Texebrionid

164. Cerandria (1).

165. Tribolium (1)

166. Boromorphus (1)

167. Calear (1).

168. 'I'enebrio (2).

169. Alphilobius (1).

Fam. 47. Opatride

170. Opatrum (2).

171. Iladrus (3).

Fim. 48. BLAPIDE.

172. Macrostelhus (1).

173. Blaps (2).

Fam. 49. Tentrriade

174. IIegeter (1).

Fam. Ј⿰0, Helopide

175. Ilelops (9).
Caxille bilobæ (lobo int simplici). Mrentum basi plerumque angustatum.

Antennce 11-art., breviuscula, apicem versus plus minusve moniliformes et incrassatx.

Corpus ellipticum vel ovatum, alatum vel apterum, plerumque glabrum, contexum, colore uetallico.

Pcdes breviusculi ; tibiis interdum spinulosis; tarsis unguiculisque simplicibus (his rariss denticulatis).

IIabitant in fungis, sub cortice arbornm laxo, vel etiam sub lapidibus; latentcs.

Iraxilla bilobre (lobo int simplici). MIcntum basi plerumque leviter angustatum.

Autenne 11-art., breves, apicem versus plus minusve moniliformes et leviter incrassatæ.

Corpus lincari-elongatum (rarius ovale), plerumque alatum, depressius culum, colore obscuro.

Pcdes longiusculi, robusti; tarsis unguiculisque simplicibus.

Mabitant in domibus, pistrinis mercatorumque rcpositoriis (prasertim inter farinas); sape commercium sequentcs.

Maxilla bilobx (lobo int plerumque simplici). Clypeus antice sæpius profunde bilobus.

Antenna 11-art., breviuscula, apicem versus plus minusve moniliformes ct vix incrassatw.

Corpus oblongum rel ovalc, apterum rel alatum, depressiusculum, interdum pilosum, colore obscuro.

Pcdes longiusculi, sat graciles; tarsis unguiculisque simplicibus.

Habitant in aridis maritimis, proscrtim sub lapidibus, vel ad graminum radices; latcntes.

Mraxille bilobx (lobo int sxpius biuncinato).

Antenne 11-art., breviuscula, apicem versus moniliformes et leviter incrassatix.

Corpus maguum, elongatum, crassum, plerumque apterum, nigr'um : clytris commatis.

Pcdes elongati; tarsis unguieulisque simplicibus.

Itabitant circa domos, vel (proeipuc in cavcrnis) per oram maritimam; luccm fugientes.

Itaxilla bilobr (lobo int sxpius simplici) : palpi max. art $t^{\circ}$ ult $t^{\circ}$ minus inflato. Mlentum amplum.

Antcuna 11-art., breriuscula, filiforınes, rel apicem versus vix incrassatio.

Corpus magnum, plus minuste crassum, plerumque apterum, nigrum; clytris sapius comatis.

Pedes elongati; tarsis unguieulisque simplicibus.

IIabitant in cavernis maritimis, vel sul lapidibus in apcrto; sese abdentes.

Maxille bilobre (lobo int sxpius simplici, obtuso). Alentum minusculun, subquadratum.

Antenne 11-art., longiusculx, filiformes, apicem versus vix sensim incrassatic.

Corpus magnum, sxpius oblongo-ovatum, convexum, alatum vel apterum ; clytris liberis rel comatis.

Pclcs clongati; tarsis antcrioribus in maribus sxpe leviter dilatatis; ungnioulis sinplicibus.

IIabitant sub lapidibus, cortice laxo, vel in eavernis; sese occultantes. 
Sectio XII. TRACHELIA

Fam. 51. OLDEMERId 176. Slenaxis (1).

Fam. 52. MELOID.E

17\%. Meloë (3).

178. Zonitis (1).

Fain. 53. MordejLID 179. Anaspis (1).

Fam. 54. Anthicida

180. Anthicus (4).

181. Xylophilus (1)

Sectio XIII. BRACHELYTRA.
Mandibula ad apicem bifidx vcl integræ, in medio sæpc fissosinuatre.

Maxillarum lobus ext ${ }^{\text {us }}$ exarticulatus ; int $t^{\text {th }}$ simplex, obtusus.

Antenne plerumque longiuscula, filiformes (rariss. pectinatx); sapius 11 -art.

Corpus plus minusve molle et laete coloratum, plerumque alatum; eapite posticc lato, truncato, in cavo prothoracico usque ad oculos liaud immerso.

Pedes terrestrii; tibiis sæpius bicalcaratis (ealeariis interdum mobilibus, æqualibus).

Tarsi hetcromeri (art. pcnultimo sæe bilobo).

Maxilla bilobæ (apicc iutcrdum longe pencillatæ) : palpi max. filiformcs, vel art ult $^{\circ}$ securiformi.

Antenne 10-12-art., longiusculæ, filiformes, vel etiam setacer.

Corpus angusto-elongatum, latc coloratum; capite porrecto; prothorace clytris (posticc subattemuatis) angustiore.

Pedes clongati femoribus maseulis sæpc incrassatis; tarsis plemumquc art $^{\circ}$ peuult ${ }^{\circ}$ bilobo ; unguieutis simplicibus.

IIabitant in Roribus; apricitate volare gaudentes.

Maxille biloba : palpi max, subfiliformes (arto ulto vix inflato).

Anterne 11-art., lougiusculx, filiformes, vel in medio incrassatre (in maribus intcrdum coutortæ).

Corpus magnum, intcrdum pictum vcl aptcrum; capite deflexo; elytris sxpe abbreviatis, complicautibus.

Pedes elongati; ealeariis sxpc inæqualibus; tarsis simplicibus; unguieulis bifidis (interdum pectinatis).

Habitant super folia plantarum humilium, pigra; vel inter arbores floresque, bene volartes.

ILaxille bilobre: palpi max. art ${ }^{\circ}$ ult ${ }^{\circ}$ plcrumquc sccuriformi.

Antenne 11-art., breviusculæ, filiformes; vel apicem versus subserratæ, pectimatro aut flabellatw.

Corpus arcuatum, pictum, subtus subcarinatum; eapite inflcxo; elytris acumiuatis, sæepc abbreviatis.

Pedes (præscrtim postici) elongati ; calcariis longis ; tarsis simplicibus ; unguieulis simplicibus vel bifidis.

Mabitant in floribus (prceipue umbelliferis); vix assultim festinantes.

Maxille bilobx: palpi max. arto ult' magno securiformi.

Antenne 11-art., breviusculæ, apiccm versus sensim incrassatæ.

Corpus parvum, plus minusvo elongatum ct pictum ; eapite pedunculato ; prothorace basi constricto.

Pedes brcviusculi, graciles; tarsis art ${ }^{0}$ penult ${ }^{\circ}$ sæpius bilobo; ungriculis simplicibus.

Habitant in graminosis et sub lapidibus, vel inter flores; sese interdum eongregantes.

Maxillarum lobus externus exarticulatus.

Antenna breviuscula, filiformes, vel leviter incrassatæ (rariss. clavatx) ; 9-11-art.

Corpus plus minusve angusto-elongatum; capite plerumque haud immerso; clytris abbreviatis (rariss. integris), abdomen magnum, durum, mobile detegentibus.

Pedes tcl'restrii ; tibiis sxepius bicalcaratis.

Tarsi plerumquc 5-art.; scd interdum 4.5 .5 ; vel omnes 4 -, ant ctiam 3-art. 
Fam. 55. SCydu ENID E 182. Scydmanus (1).

Fam. 56. StapiryunId A.....

183. Falamria (1)

184. Tachyusa (1)

185. Xenomma (3).

186. Ilomalota (15).

187. Oxypoda (1).

188. Aleochara (4).

189. Oligota (1).

190. Somatinm (1)

191. Conurus (3).

192. Tachyporus (2)

193. Ilabrocemus (1).

194. Tachinus (1).

195. Trichophya (1)

196. Mycetoporns (1)

197. Othius (2)

198. Xantholintes (2)

199. Staphylinus (1).

200. Philonthus (7).

201. Achenium (1)

202. Lathrobium (1).

203. Lithocharis (3).

204. Rugilus (1).

205. Sunius (2).

206. Mecogmathus (1).
JLaxilla bilobre. Palpi arto ult ${ }^{\circ}$ minutissimo, subulato.

Antenna 11-art., longiuseulæ, sensim elavatæo (clavâ laxâ, 3- vel 4-art.).

Corpus ninutum, oratum; protleorace basi constrieto; elytris abdomen totum tegentibus.

Pedes longiuseuli, graeiles.

Tarsi 5-art., simpliees. Habitant in graminosis, cultis, vel inter muscos; interdum una cum for-
mieis degentes.

(Naxille bilobre. Palpi art ${ }^{0}$ ult ${ }^{0}$ vel elongato, vel parro subulato (rariss. seeuriformi)

Antenna sæpius 11- (rarius 10-, rariss. 9-) art., filiformes vel leviter inerassatx, interdun geniculatx.

Corpus elongatum (rarius ovatum); prothorace vel elstronum latitudine, vel iis (abbreviatis) vix angustiore. Pedes longiuseuli vel breviuseuli ; (anteriores plerumque paulo breviores,
ralidiores).

Tar'si 3-5-art.; rel ant $t^{i}$-, et postes 5-art.; (sed plerumque omnes 5-art.). Habitant in quisquiliis, per margines aquarum, vel in stcreore; scepius
valde voraces.

Subf. 1. Ateocinarides.

Mandibula srepius mutiex. Palpi max. art ${ }^{\circ}$ ult ${ }^{\circ}$ parvo, subulato.

Antenna 11- (rarius 10-) art., ad oculorum marginem internum insertx, reetx, subfiliformes.

Ligula angusta, porreeta, plerumque apice bifida.

Corpus parvum, sæpius lineare, depressiuseulum; labro integro.

Tar'si 5- (rarius 4-) art.; vel antici 4-, et posteriores 5-art.: (antici nomumquam dilatati).

Subf. 2. TACHrPorides.

ILandibula sxpius muticx. Palpi max. art ${ }^{0}$ ult ${ }^{0}$ rel parro subulato, rel precedente aquali.

Antenna 11- (rarius 10-) art, infra oeulos sub frontis margine insertæ, rectæ, subfiliformes.

Ligula lata, plerumque biloba.

Corpus parvum, sxpius fusiforme, eonrexiuseulum; labro integro.

Tibica (vel omnes, vel posteriores soluru) sæpius spimulosæ.

Tarsi 5- (rarius 4-) art.: (antici sxpe dilatati).

Subf. 3. Stapurimines.

Mandibula sæpius medio dentatx. Palpi max. art ${ }^{\circ}$ ult ${ }^{\circ}$ praeedente subrequali. Antenna 1l-art., in frontis margine anteriore insertre, sape genieu-
latre et leriter inerassatæ.

Ligula parra, biloba rel integra.

Corpus plerumque magnum, lineare, depressiuseulum; labro bilobo.

Tibice (rel ommes, rel posteriores solum) sæpius spinulosie.

Tarsi 5-art.: (antiei, prasertim in maribus, sæpe dilatati).

Subf. 4. Paderides.

Mandibula tenues, elongatr, medio dentate. Palpi max. arto ult ${ }^{\circ}$ minuto, sæpius subulato.

Antennce 11-art., infra oeulos sub frontis margine inserte, plerumque recte, filiformes.

Ligula biloba, lobis modo approximatis modo distantibus.

Corpus parvuseulum, sapius angusto-filiforme; labro bilobo, vel bidentato.

Prothorax immarginatus. Scutellum distinetum, triangulare.

Tarsi 5-art. : (antici interdum dilatati). 
207. Stenus (4).

208. Platysthetus (2).

209. Oxylelus (5).

210. Trogophloeus (1).

211. Omalium (2).

212. Megarthrus (1) 213. Metopsia (1).
Subf. 5. Stenides.

Mandibulce tenues, clongatr, pone apicem valde unidentatx.

Palpi max, art ${ }^{\circ} 1^{\circ}$ elongato, ult ${ }^{\circ}$ minutissimo (vix observando).

Antennœ 11-art., inter oculos (in froute) plerumque insertæ, rectæ, clavatæ.

Ligula levissime mento affixa (quare, insecto moriente, cum œsophago sæpe prolabitur).

Corpus parrusculum, filiforme; capite magno; labro integro vel denticulato.

Prothorax immarginatus. Scutellum vix distinctum. Coxa antiece minutæ.

Tarsi 5- (rarins 4-) art., graciles.

Subf. 6. Oxtrelides.

IFandibule validiores, sæpius dentatæ. Palpi max. art ult plerumque subulato.

Antenne 11- (rarius 10-) art., sub frontis margine laterali insertx, sæpe refractse, snbiucrassatre.

Ligula apice sinuata, vel biloba (rarius integra).

Corpus parvum, lincare, subcylindricum vel depressum; labro membranâ utrinque acuminatî aucto.

Tarsi 3- (in spcciebus aberrantibus 5-) art, plerumque graciles.

Subf. 7. Omalines.

Mandibule breves, sxpins muticx. "Palpi max. filiformes, art ult $^{\circ}$ longiusculo.

Antenna 11-art., sub froutis margine latcrali inserta, recta, apicem versus vix incrassitx.

Ligula lati, biloba. Maxillarum lobus internus unco corneo armatus. Corpus parrum, lineari-oblongum, depressnm ; fronte occllis duobus iustructâ.

Elytra pectore longiora, angulis exterioribus apicalibus rotundatis.

Tarsi 5-art.: (antici rains subdilatati).

\section{Subf. S. Proteinides.}

Mandibula breves, muticse. Palpi max. filiformes, arto nlto longiusculo.

Antenne 11- (rurius 9-) art., sub frontis margine laterali insertæ, rectx, subclavatx.

Ligula biloba (rariss. integra). Maxillarum lobus internus nnco (interdum duplice) armatus.

Corpus parvum, siepius ovatum, latiusculum, depressum ; fronte rarius ocello instructâ.

Elytra pectore longiora. Coxa antiec cylindrica, haud exserta.

Tursi 5- (vel 3-) art., breviuseuli. 


\section{CAT A LOGUS TOPOGRAPHICUS.}

\section{Seetio I. GEODEPHAGA.}

Fam. 1. Carabidæ.

(Subf. 1. Brachinides.)

1. Tarus, Clairv.

1. lineatus, Schön.

2. suturalis, $D e j$.

2. Dromius, Bon

3. insularis, Woll.

4. $\{$ sigmn, Rossi,

6. obscurog

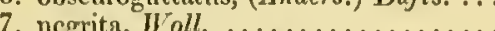

8. glabratus, (Meg) Dufts. .............

\section{(Subf. 2. Scaritides.)}

3. Scarites, Fab.

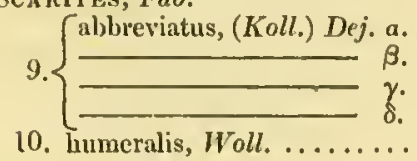

4. Аротомиs, Hoffm.

11. rufus, Rossi .

\section{(Subf. 3. Caralides.)}

5. Calosoma, Weber

12. Maderæ, Fab.

6. Notiophilus, Dum.

13. geuninatus, $D e j$.

\section{(Subf. 4. Harpalides.)}

(Div. 1. Chlaniidea.)

7. Loricera, Lat.

14. Wollastonii, Javet

8. Eurygnathus, Woll.

15. $\{$ Latreillci, Lap........

9. ZARGUS, Woll.

16 Schaumii, Woll.

$17^{\circ}$ Deserta, $/{ }^{\prime}$ oll .

18. $\{$ pellucidus, Woll. $\ldots$.

10. Pristonycilles, Dej.

(Div. 2. Pterostichidea.)

19. alatus, Holl.

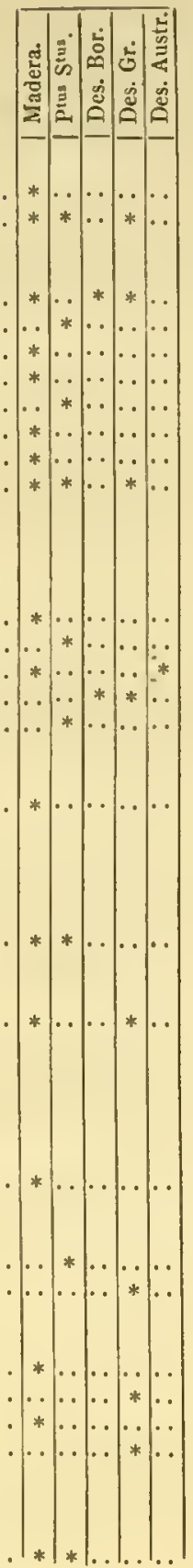

11. Calathus, Bon.

20. vividus, $F a b$

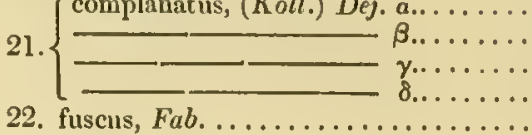

12. ANCHOMENUS, Bon.

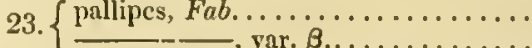

24. marginatus, Linn.

13. Olisthopus, Dej.

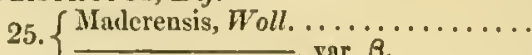

26. Erice Holl , var. $\beta . . . . . . . .$.

27. elongatus, Woll. ..............

14. Argutor, (Meg.) Steph.

28. robustus, Woll.

29. gracilipes, Woll.

30. dilaticollis, Woll.

31. $\left\{\right.$ curtus, $W_{\text {oll }}$, var.

15. Omaseus, (Ziegl.) Steph.

32. nigerrimus, $D e j$.

33. Wollastoni, Heer

16. AMARA, Bon.

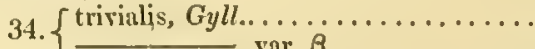

35. superans, Woll.

17. Anisodactyuus, Dej.

(Div. 3. Harpalidea.)

36. binntatus, $F a b$.

18. IArpalus, Lat.

37. $\{$ attcmintus, Steph............

38. litigiosus, Dej , vur. $\beta . \quad \ldots \ldots$.

39. distingneudus, Dufts..............

40 $\{$ vividus, $D e j$. a. . . . . . . . . . .

40. $\left\{\begin{array}{l}\text { vividins, Dej. } \\ -\beta . \\ -\gamma \text {. }\end{array}\right.$

19. Opuonus, (Ziegl.) Steph.

41. obseurus, Fab.....

20. Stenolophus, (Meg.) Steph.

42. Teutonus, Schr

43. dorsnlis, Fal.

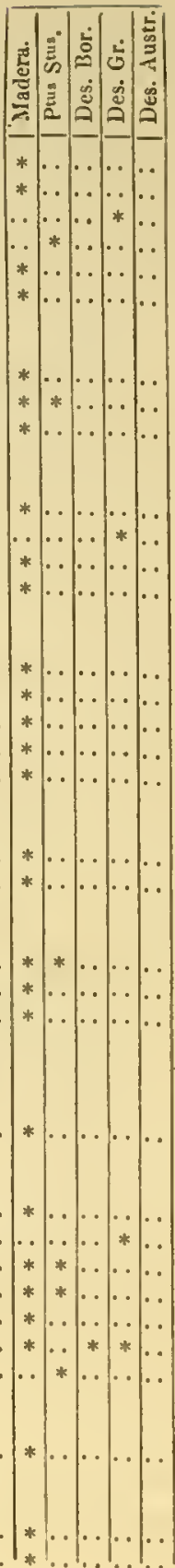


21. Bradycellus, Erich.

44. fulvus, $M s h m$

45. \{

$\{$ excultus, Woll. $\ldots . .$.

22. Trecirus, Clairv.

46. fimicola, Woll.

47. $\{$ nigrocruciatus, Woll............

$\{7$, var. $\beta$

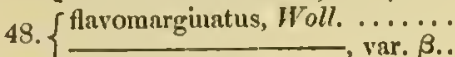

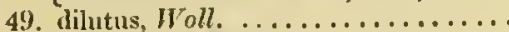

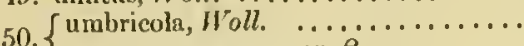

$50 .\{\ldots$ var. $\beta . . . \ldots \ldots$

51. quadricollis, Woll. ...............

52. custos, Woll.

53. alticola, Woll.

54. cautus, Woll.

23. Tilalassophilus, Woll.

55. Whitci, Woll.

(Subf. 5. Bembidiades.)

24. Bembinius, Lat.

56. bistriatum, (Meg.) Dufts.

57. curvimanum, Woll

58. Lucasii, Duval .

59. obtusum, Sturm

....................

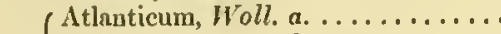

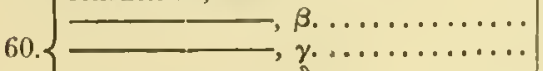

(

61. tabellatum, Woll. ............... *

62. elongatum, $D e j . . . \ldots \ldots \ldots \ldots \ldots \ldots$ *

63. Selımidtii, Woll.

\section{Sectio II. IHYDRADEPHAGA.}

Fam. 2. Dytiscidæ.

25. Colyabetes, Clairo.

64. Lanio, Fab. ..

26. AGa Bus, Leach

65. bipustulatus, Linn. ............

66. $\{$ nebulosus, Forst. $\ldots \ldots \ldots \ldots \ldots \ldots$

67. Maderensis, Woll. , . . . . . . . . .

27. Hrdmoponus, Clairv.

68. vigilans, Woll.

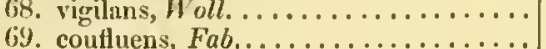

Fam. 3. Gyrinidx.

28. GyrINUS, Linn.

70. natator, Linn.

\section{Sectio III. PHILHYDRIDA.}

Fain. 4. Parnidæ.

29. Parnus, Fab.

71. prolifericornis, Fab.
Fam. 5. Hydrophilidæ.

30. Ochтmini us, Leach

72. 4-foveolatus, (Mots.) Woll.

31. Calobius, Woll.

73. IIecri, Woll.

32. LIMNEBIUS, Leach

74. grandicollis, Woll.

33. Laccobius, Erich.

i5. minutus, Linn

34. HYDRoBI Us, Leach

76. couglobatus, Woll.

35. Piilliyprus, Sol.

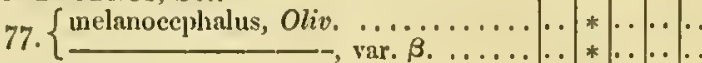

Fam. 6. Sphæridiadæ.

36. Dacty losternum, Woll.

78. Roussctii, Woll.

37. SPI KEIDIUм, Fab.

79. bipustulatum, Fab.

38. CERCYon, Leuch

80. inquinatum, Woll

81. fimetaium, Woll.

82. $\{$ centrimnculatum, Sturm

83. quisquilium, Linn.

\section{Seetio IV. NECROPHAGA.}

Fam. 7. Silphidæ.

39. Catops, Payk.

84. velox, Spence

Fam. 8. Ptiliadæ.

40. Acratricilis, Mots.

85. umbricola, Woll.

86. fuscicularis, Herbst

87. pumila, Frich....

41. P'Tenidium, Erich.

88. npicale, (sturm) Gilln.

Fam. 9. Phalacridæ.

42. Oนin Rus, Erich.

89. Cincratia, Woll. ............

90. bicolor, Fab....................

91. liquidus, Erich. ..............

92. consimilis, $M \mathrm{shm}$

Fam. 10. Nitidulidæ.

43. Canpopirilus, (Leach) Stcph.

93. mutilatus, (IIoffin.) Ericle.

94. auropilosus, Holl.

95. heminterus, Linn.

44. Nitidula, Fab.

96. Hexnosa, Oliv.

97. 4-pustulata, $\mathrm{Fab}$

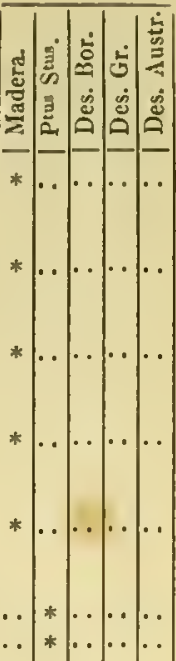

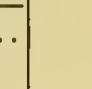


98. discoidea, $F a b$

99. obsoletr, Fab.

45. Pria, (Kirby) Steph.

100. Duleamara, Scop

46. Meligeties, (Kirby) Steph.

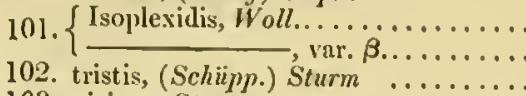

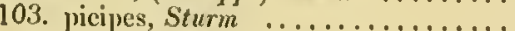

104. $\{$ varicollis, Woll. ...........

47. Xenostrongyuus, Woll.

]05, histrio, Woll. .....

Fam. 11. Colydiadæ.

48. Tariuius, (Germ.) Erich.

106. parallelus, Woll...

107. Lowei, Woll.

109. spinipes, Woll.

110. sylvicola, Woll.

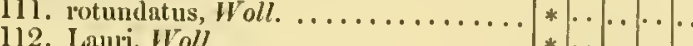

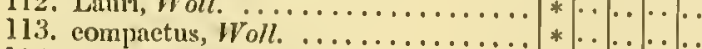

114. molosus, $V_{0}$.

115. cieatricosus, Woll. . . . . . . . . . .

116. testudinalis, Woll. . . . . . . . . .

117. truncatus, Woll...............

118. chinatus, Woll

119. brevicollis, Woll . ............

120. rugosus, Woll. .............

49. Cossrpuodes, Westw

121. Wollastonii, Westw.

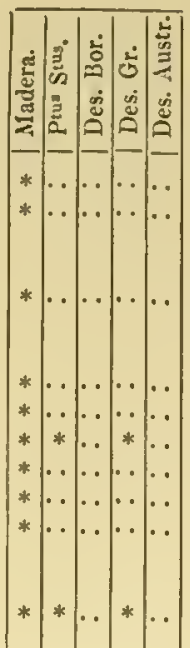

56. Silvanus, Lat.

136. Surinamensis, Linn.

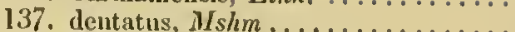

138. advenn, (Kunze) Waltl.

Fam. 14. Cryptophagidæ.

57. Сиy рторhagus, Herbst

139. affinis, Sturm ....

483. Nitiduloides, Woll.

58. Dipiyulus, Redt.

140. lunatus, $\mathrm{Fab}$

59. Hypocoprus, Mots.

141. Motschulskii, Woll.

60. Epilistemus, (Westw.) Steph.

142. dimidiatus, Sturm
143. alternans, IVoll.

Fam. 15, Lathridiadæ.

61. Cholovocera, Mots.

144. Madera, (H'estw.) Woll

62. Holoparamecus, Curtis

145. niger, (Chevr.) Aubé

63. Corticaleia, Mshm

146. rotulicollis, W'oll.

147. erenicollis, Mann.

148. fulva, (Chevr.) Mann.

149. rotundicollis, $W^{*}$ oll.

150. curta, Iroll.

151. Fagi, Woll

64. Latimidius, Herbst

152. assimilis, Mann

153. minutus, Linn.

154. transversus, Oliv.

65. Mrtopitialuus, (Mots.) Woll.

155. asperatus, Woll.

Fam. 16. Mycetophagidæ.

66. Berginus, (Dej.) Erich.

156. 'Tamarisci, (Dej.) Woll.

67. Microchondous, (Guér.) Holl.

157. domuum, (Guér.) H'oll.. .

53. Trogosita, Oliv.

125. mauritanica, Linn.

126.

68. Турнжа, (Kïirby) Steph.

158. fumata, Linn

Fam. 13. Cucujidæ.

54. CRy ptamonpha, Woll.

127. Muse, Holl.

55. Lamophugeus, (Dej.) Erich.

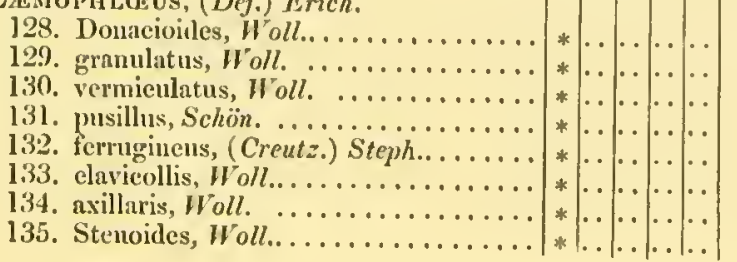

69. Litargus, Erich.

159. pictus, Woll

Fam. 17. Dermestidx.

70. Dermestes, Jinn.

160. vulpinus, Fab.

71. ATtageNus, Lat.

161. megatoma, Fab.

72. Anthrenus, Geoffr.

162. varius, Fub.

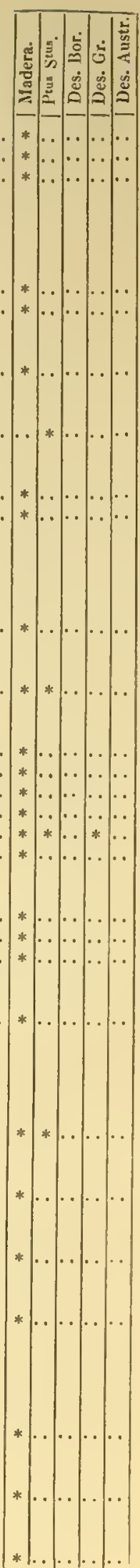




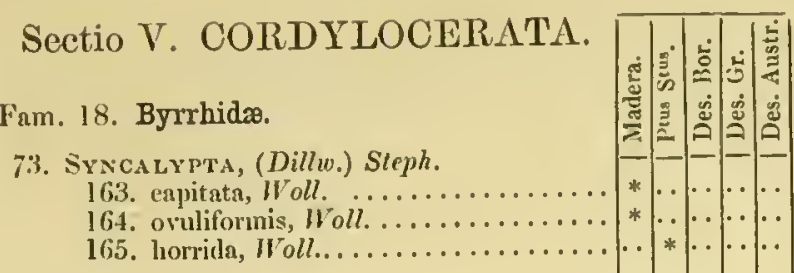

Fam. 19. Histeridæ.

74. Hister, Linn. 166. major, Linn.

75. Paromalus, Erich. i 67. minimus, (Dej.) Aubé 168. pumilio, Erich.

76. SAPRINUS, Erich.

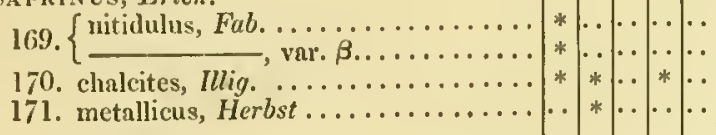

Fam. 20. Thorictidæ.

77. Thorictus, Germ.

172. Westwoodii, Woll.

Fam. 21. Aphodiadæ.

78. APноDius, Illig.

173. Hydrochæris, Fab.............. * *....

174. nitidulus, $F a b$.

175. rufus, Illig..

176. lividus, Oliv.

177. Pedrosi, Woll. ......................

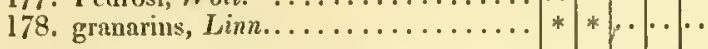

79. Oxyosus, (Esch.) De Casteln.

179. Heineckeni, Woll. .....................

180. brericollis, Woll. .................

80. Psammodius, Gyll.

181. saluulosins, (Dej.) Mulst

182. cxesus, $P n z$.

Fam. 22. Trogidæ.

81. Trox, Fab.

183. scabcr, Linn. .

Fam. 23. Glaphyridæ.

82. Chasmatopterus, (Dej.) Lat.

184. nigrocinctus, Woll.

\section{Sectio VI. PRIOCERATA.}

Fam. 24. Throscidæ.

83. Trixagus, Kugell.

185. gracilis, Woll.

Fam. 25. Elateridæ.

84. Coptostetiues, Woll.

186. femoratus, Woll.

Fam. 26. Cyphonidx.

85. Eucinetus, Schiipp.

187. ovum, Woll.

\begin{tabular}{llllll}
$*$ & $*$ & $\cdots$ & $\ldots$ & $\cdots$ \\
$*$ & $\ldots$ &. & $\ldots$ & $\ldots$ \\
\hline
\end{tabular}
Fam. 27. Telephoridæ.

86. Maltuodes, Kies.

188. Kicsenwetteri, Woll.

Fam. 28. Melyridæ.

87. Malachus, Fub.

189. militaris, Woll.

88. Pecteropus, Woll.

190. $\{$ Maderensis, Woll. $\ldots \ldots \ldots \ldots \ldots$. $\dddot{\beta} \ldots \ldots \ldots$

191. mgosus, Woll. ................

192. $\{$ rostratus, Woll, a

89. DASY'TES, Payk.

193. illustris, (Mots.) Woll.

90. MELYROSOMA, Woll.

194. occanienm, Woll.

195. Artcmisix, Woll.

Fam. 29. Cleridæ.

91. Opizus, Lat.

196. mollis, Linn.

92. Necrobia, Oliv.

197. ruficollis, Theng.

Fam. 30. Ptinidæ.

93. Ptisus, Limn

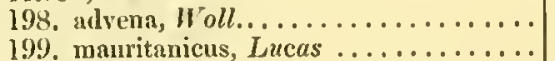

Dow Woll.

201. pinguis, Woll.

202. orbatns, Woll..

203. nodulus, Woll.

204. pilula, Woll.

albopictus, Woll a.

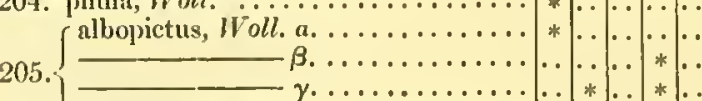

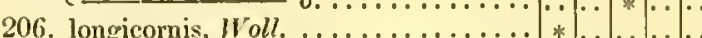

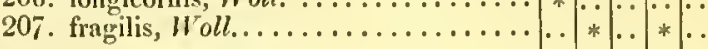

94. MEzi un, (Leach) Curtis 208. sulcatum, $\mathrm{Fab}$.

95. Giввím, Scop.

209. scotias, $F a b$.

96. Aлович, Fab.

210. velatum, Holl.

211. panicenm, Lin

212. striatnm, Oliv.

213. Ptilinoides, Woll. .... ..........

Fam. 31. Cissidx

97. Cis, Lat.

214. Wollastonii, Mellié

215. fuseipes, (Chevr.) Mellie ............

216. Lauri, Woll.

98. Octotemnus, Mellié

217. opacus, Mellié

99. P'rilinus, Geoffr.

218. cylindripenuis, Woll.

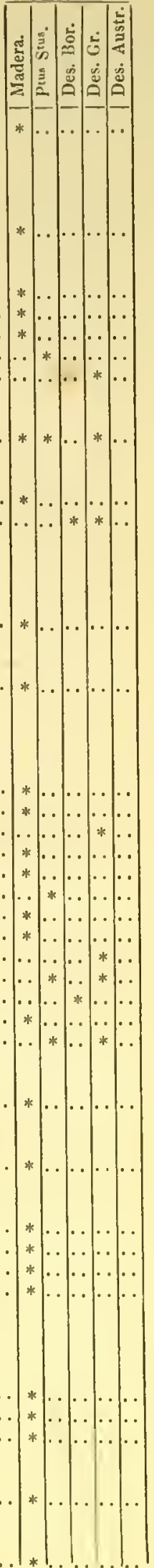




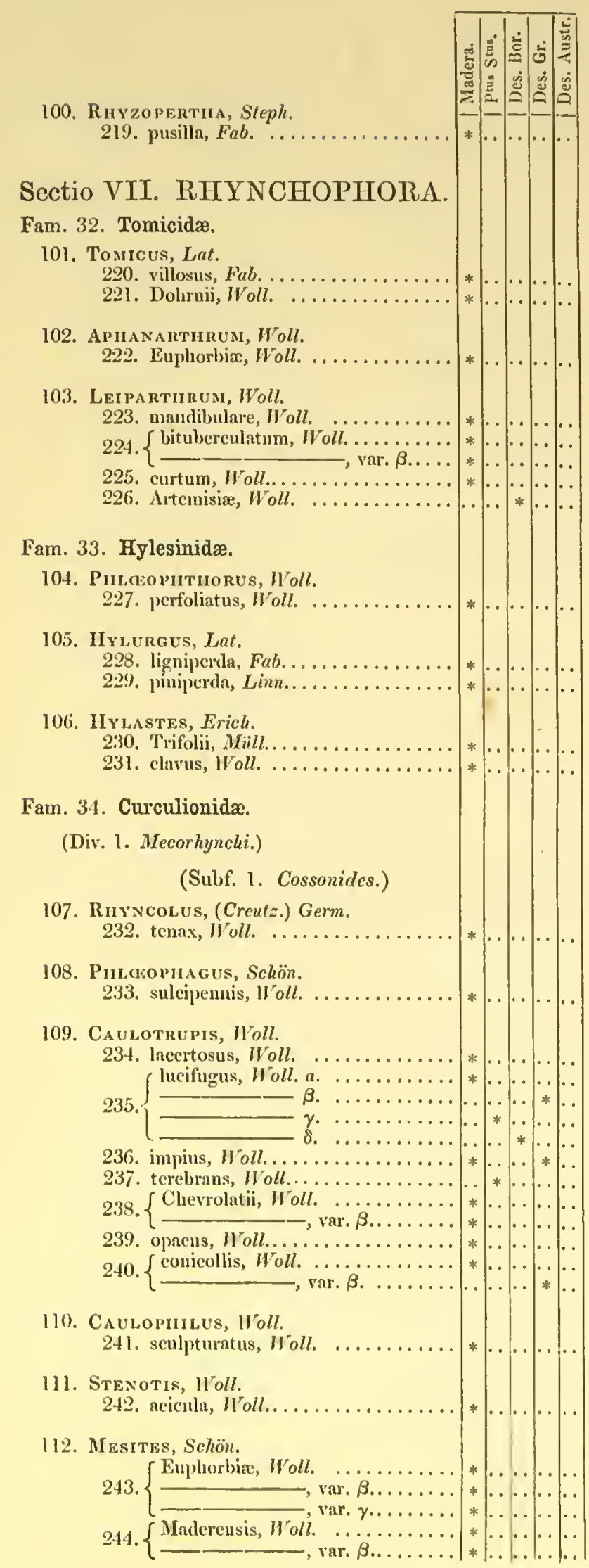

(Subf. 2. Rhynchophorides.)

113. Sitophulus, Schön.

245. granarius, Lin

(Subf. 3. Cionides.)

114. Cronus, Clairv.

247. pulchellus, Herbst

(Subf. 4. Cryptorhynchides.)

115. Ceutorirycilus, (Schïpp.) Schön.

248. Echii, Fab.

249. quadridens, $P_{n z}$

250. nigroterminatus, Woll

251. lineatotesscllatus, Woll...........

116. Cathodes, Schön.

252. fuliginosus, Mshm .

117. ACALLES, Schön.

253. saxicola, IVoll.

254. pulverulcutus, Woll....................

255. oblitus, Noll.

256. nodiferus, Woll.

257. Vau, Holl.

258. $\{$ terminalis, Woll. .............

259. ornatus, Woll..............

260. dispar, Holl. . .

261. albolineatus, Woll.

262. globulipennis, IVoll.

263. lunnlatus, Woll.....

(Subf. 5. Erirhinides.)

118. Tycuies, (Germ.) Scliön.

266. robustus, $\mathrm{Woll}$.

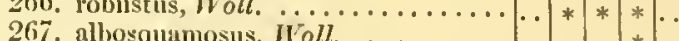

268, filirostris, II'oll.

119. Pissodes, Germ

269. notatus, $F a b$.

120. Lixus, Fab.

270. Cheiranthi, Woll.

271. Chawneri, Holl.

$\ldots \ldots \ldots * * *+*$

272. rectiformis, Iloll. .............

273. angustatus, Fub.

274. rutitarsis, Schön.

(Div. 2, Braclyyrhynchi.)

(Subf. 6. Cyclomides.)

121. Cyphoscelis, Woll.

275. distorta, Holl..

122. Liparocerus, Schön.

276 . moriu, Schön.

123. Atrantis, Woll.

277. claratus, IVoll.

278. lamellipes, Ifoll.

279. calcatrix, Woll.

280. noctivagaus, Wol

281. lauipotens, I'oll

282. australis, IIoll.

283. respertims, IIoll. ...........

284. lamatus, Woll.. 


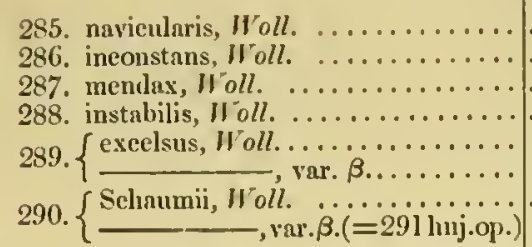

124. Omias, (Germ.) Schön.

292. ventrosus, Woll.

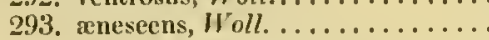

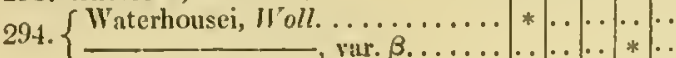

125. ANEMOPHILUS, Woll.

295. erassus, Woll. ........................

296. subtessellatus, Woll...................

297. trossulus, Woll.

126. Lichenopilagus, Woll.

298. fritillus, Woll..

299. acuminatus, Woll.

127. Scoliocerus, Woll.

300. Madera, Woll.

301. eurvipes, Woll

128. Trachyphloevs, Germ.

302. scaber, Linn.

(Subf. 7. Byrsopsides.)

129. Echinosoma, Woll.

303. porcellus, $W$ oll.

(Subf. 8. Molytides.)

130. IY PERA, Germ.

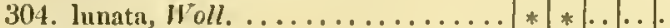

305. murina, Fab. ....................

306. variabilis, Herbst ...........

(Subf. 9. Cleonides.)

131. Cleonus, Scliön.

307. plieatus, Oliv.

(Subf. 10. Bruchyderides.)

132. Sitosa, Germ.

308. gressoria, $F a b$.

309. latipennis, Schön.

310. eambrien, $(K b y)$ Steph.

311. lineata, Linn..........

Fam. 35. Attelabidæ.

133. Apros, Herlst

313. vernale, $\mathrm{Fab}$

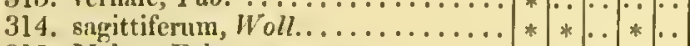

315. Malve, Fub. .....................

316. frumentarium, Linn............ * * ......

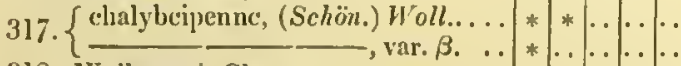

318. Wollastoni, Cherr..............

319. rotundipeune, Woll. .............
134. Avletes, Schön.

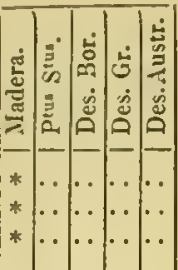

\section{Fam. 36. Bruchidæ.}

(Subf. 1. Anthribides.)

135. Xenorchestes, Woll. 321. saltitans, Woll.

(Subf. 2. Bruchides.)

136. Bruciuvs, Gcoffr.

322. nutimanus, Schön....................

323. subellipticus, Woll.

324. lichenieola, Woll.

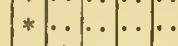

\section{Sectio VIII. EUCERATA.}

Fam. 37. Cerambicidæ.

137. Stromatium, Sero.

325. unicolor, Oliv..

138. Риymatodes, Mulst

326. $\{$ variabilis, Linn. $\ldots \ldots$.

139. Buabinotus, Woll.

327. spinieollis, Woll.

140. Trichoferus, Woll.

328. senex, Woll.

141. Clytus, Fab.

329. Arietis, $\operatorname{Linn} . . . \ldots \ldots \ldots \ldots \ldots \ldots$. . . . . . . . .

142. DevCALION, Woll.

330. Desertarum, Woll.

\section{Sectio IX. PHYTOPHAGA.}

Fam. 38. Crioceridæ.

143. Lesa, $F a b$.

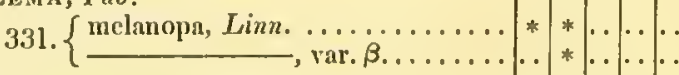

144. CHOCERIS, Geoffr.

332. Aspatngi, Linn.

Fam. 39. Cassididæ.

145. Cassion, Linn.

333. nelulosa, I,inn.

334. lemisplaterien, Herbst

Fam. 40. Galerucidæ.

146. Inatica, Geoflr

335. subtilis, Woll................**...*..

336. Salicarixe, Payk. ............ *

147. Longitareus, Lat.

3:37. Isoplexirlis, I"oll. . . . . . . . . * *

338. Cincraria, I'oll. ...........*

339 . saltator, IVoll. 
340. luteseens, Gyll.

341, \{ nervosus, Woll...............

342, nubigena, Holl, var........

148. Psylliones, Lot.

343. chrysocephala, Linn

344. hosises, Woll.

45. umbratilis, Woll ..........

346. $\{$ vehemens, Woll.,$\ldots \ldots$

37. taruatin, WToll.

Fam. 41. Chrysomelidæ.

149. Mniophilosona, Woll.

348. lave, Woll. . . . . .

150. Cryptocen'ila lus, Geaffr.

349. crenatus, 1 oll.

151. Chrysomfa, Linn.

350. Fragarix, Woll.

152. Gastropliysa, (Chevr.) Redt.

351. Polygoni, Linn. ........

\section{Sectio X. PSEUDOTRIMERA.}

Fam. 42. Coccinellidæ.

153. Coccinella, Linn.

352. mutabilis, Scriba

354. 14-pustulata, Linn.

355. $\{$ testudinea, (Hein.) Woll........

356. Genista, $W_{\text {oll }} \ldots \ldots \ldots \ldots \ldots \ldots \ldots$

154. Sicrmus, Kugell.

$357 .\left\{\begin{array}{l}\text { Duranta, } W^{r} \text { oll. } \ldots \ldots \ldots \ldots \ldots \\ \text { var. } . \ldots \ldots \ldots\end{array}\right.$

358. $\{$ luarginalis, Rossi ...........

arcuatus, Rossi, a. ...........

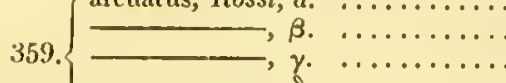

ภ. $\cdots \ldots \ldots \ldots \ldots$

.

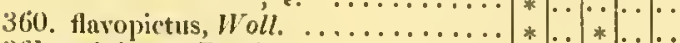

361. minimus, Rossi ............. * .......

362. Limnichoiles, $\|$ oll. . . . . . . . . $* \ldots \ldots \ldots \ldots$

155. Rиyzoвrus, Stepl. $363 .\left\{\begin{array}{l}\text { litura, } F a b . \ldots \ldots \ldots \ldots \ldots \ldots * \ldots \ldots \ldots \ldots \\ \text { var. } \beta . \ldots \ldots \ldots\end{array}\right.$

Fiur. 13. Corylophidx.

156. Chy'lisaren, (Anders.) Redt. 3ijt. pusillus, Gyll..........

15\%. ARTUROL1PS, II oll.

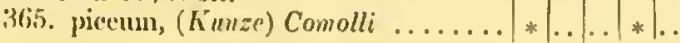

158. Sericonkrus, Steph. 366. lateralis, (Meg.) Gyll.

159. Coryloryus, (Leoch) Steph. 367. tectiformis, IYoll.

160. GLa:osoma, Woll. 368. velox, Woll.

\section{Sectio XI. A'TRACHELIA.}

\section{l'am. 44. Anisotomidæ.}

161. Sta gonomorpha, Holl.

369. spligerula, Holl.

370. unicolor, $W^{\prime}$ oll.

\section{Fam. 45. Diaperidæ.}

162. Ellipsodes, Woll.

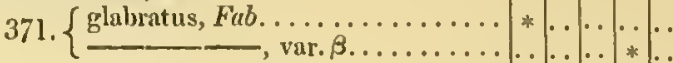

163. Phaleria, Lat.

372. ciliata, II oll.

Fam. 46. Tenebrionidæ.

164. Cerandria, (Dej.) Lucas 373. cornuta, Fo

165. Trinolium, MocLeoy

374. fcrrugineum, $F a b$.

166. Boromorphus, (Mots.) Woll. 375. Maderie, 1 oll.

167. Calcar, (Dej.) Lot. 376. clongatus, Herbst

168. TENEBRIO, Linn.

377. molitor, Limn

378. obscurus, Fab.

169. Alphitobius, Steph. 379. diaperinus, Kugell.

Fam. 47. Opatridx.

170. Opatrum, Fab.

380. fuscunı, Herbst ............... * * ...*. 381. crrans, I $^{\circ}$ ll.

171. Hadrus, (Dej.) Woll.

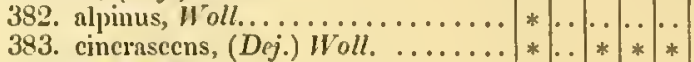
384. illotus, Itoll

Fam, 48. Blapsidæ.

172. Macrostetues, Woll. 385. tuberculatus, Woll

173. BLAps, Fob.

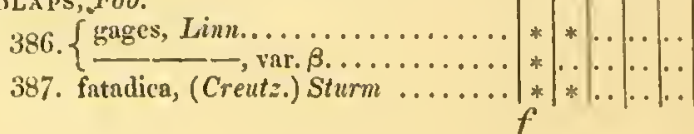


Fam. 49. Tentyriadæ.

174. Hegeter, Lat.

388. elongatus, Oliv.

Fam. 50. Helopidæ.

175. HeLOH' Fab.

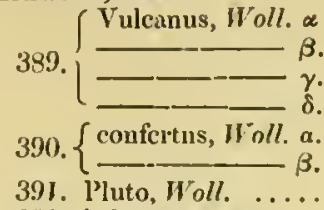

392 iuferuus, IVoll.

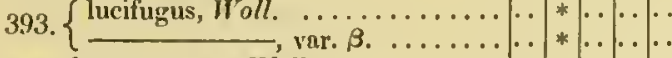

394. $\{$ congregatus, Woll. $a . \ldots \ldots \ldots \ldots \ldots \ldots * \ldots$.

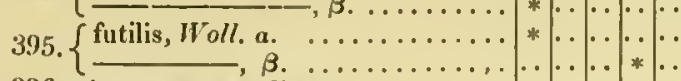

396. cinnamomens, Woll. . . . . . . . * . . . . .

397. Portosanctamis, Woll.

\section{Sectio XII. TRACHELIA.}

Fam. 51. Edemeridx.

176. Stenaxis, Schmidt

398. Lowei, Woll.

Fam. 52. Meloidæ.

177. MELö̈, Linn.

399. austrinus, Woll.

400. rugosus, $M f s h m$. . . . . . . .

$\ldots \ldots \ldots \ldots$

178. Zovit1s, Fab.

402. $\{$ 4-punctata, Fab. ............

Fam. 53. Mordellidæ.

179. Anaspis, Geoffr.

403. $\{$ Protcus, Woll. ..............

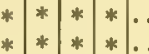

Fam. 54. Anthicidæ.

180. Antricus, Payk.

404. instabilis, (Hoffm.) Schmidt ......

405. litoralis, Heer................. 406. hispidus, Rossi ................... 407. $\{$ tristis, Schnidt...$\ldots \ldots \ldots \ldots$

181. Xylophilus, (Bonelli) Lat. 408. pallescens, Woll.

\section{Sectio XIII. BRACHELI'TRA.}

Fam. 55. Scydmænidæ.

182. SсYрм $A$ NUs, Lat. 409. Helfuri, Schaum
Fam. 56. Staphylinidæ.

(Subf. 1. Aleocharides.)

183. FA La Gind, (Leach) Mamn.

410. obscura, Grav.

184. TAchYUsa, Erich.

411. raptoria, Woll.

185. XENOMMA, Woll.

412. planifrons, Woll.

413. formicarum, Wol

414. filiforne, Woll.

186. Hом а LoTA, Mann.

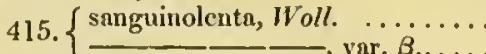

416. granulosa, Woll...............

417. obliquepunctata, Woll. ..............

418. luticola, Woll...

419.

420. Philonthoides, Woll. . . . . . . . .

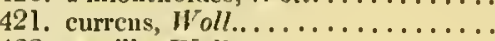

422. tantilla, Woll.................

423. plebcia, Woll ...................

424. sodalis, Erich.

425. umbratilis, Wo

426. insignis, Woll.

427. atranentaria, (Kby) Gyll.......

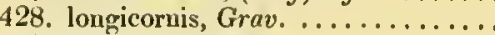

429. lividipcunis, Mann.............

187. OXY1'ODA, Mann.

430. litigiosa, Heer

188. Aleochara, Grav.

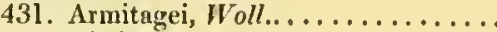

432. tristis, Grav.

133. $\{$ nitida, Grav.

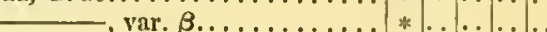

434. morion, Grav.................. * *

189. Oligota, Mann.

435. inflata, Mann

(Subf. 2. Tachyporides.)

190. Somatium, Woll.

436. anale, Woll.

191. Conurus, Steph

437. puheseens, Payk. ................ * *

438. pedicularius, Grav............. * * ...

439. $\{$ monticola, Woll. ............ * * . . .

192. Tachyporus, Grav.

440. celer, Woll. .

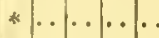

193. Habrocenus, Erich.

442. capillaricoruis, Grav.

194. Tachines, Grav.

443. Silphoides, Linn.

195. Triciophy, Mann.

444. Iluttoni, Woll.

196. Mrcetoporus, Mann.

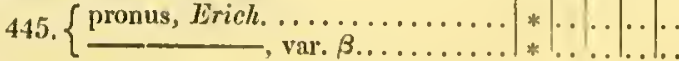


(Subf. 3. Staphylinides.)

197. Ormius, (Leach) Steph. 446. strigulosus, Woll. 447. Jansoni, IVoll.

198. Xantrolinus, Dall 448. punctulatus, Payk 449. linearis, Oli

199. Staphylinus, Linn. 450. maxillosus, Lizn.

200. Philonthus, (Lcach) Stcph.

451. xneus, Rossi

452. umbratilis, Grav.

453. sordidus, Grav.

454. bijustulatus, $P$ n

455. varians, $P y k$.

456. aterrimus, Grav.
457. filiformis, Woll.

(Subf. 4. Paderides.)

201. Achenum, (Lcach) Curtis. 458. Hartungii, Heer

202. Lathrobium, Grav.

459. multipunctatum, Grav.

203. Lithocharis, (Dej.) Lacord. 460. fuscula, (Zicgl.) Lacord.

461. ochracen, Grav....

............

462. melanocc|lıala, $\ddot{F} a b$

204. Rugilus, (Lcach) Curtis 463. affinis, Erich.

205. Sunius, (Leach) Steph.

464. angustatus, Payk.

465. bimaculatus, Ericli.

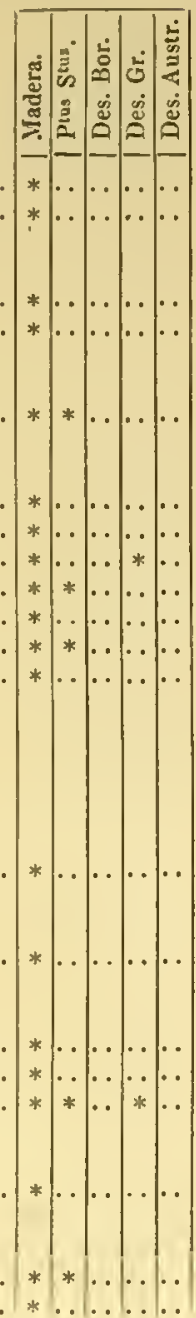

206. Mecognatius, Woll. 466. Climæra, Woll.

(Subf. 5. Stenides.)

207. Stenus, Lat.

467. guttula, Müll.

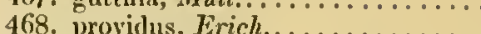

409. undulatus, Woll. $470 .\{$ Hecri, Woll. . . . . . . . . . . . . . .

(Subf. 6. Oxytelides.)

208. Platystietus, Mann.

471. spinosus, Erich.

472. fossor, Woll.

209. Oxytelus, Grav.

473. piceus, Linn.

474. sculptus, Grav.

475. complanatus, Erich

476. nitilulus, Grav.

477. glareosus, Woll.

210. Trogophlueus, Mann. 478. nanus, Woll.

(Subf. 7. Omaliades.)

211. Omalium, Grav.

479. ocellatum, Woll

480. granulatum, Woll

(Suljf. 8. Proteinides.)

212. Merartirus, (K'by) Steph. 481. longicornis, Woll. . .

213. Mrfopsia, Woll.

482. ampliata, Woll.

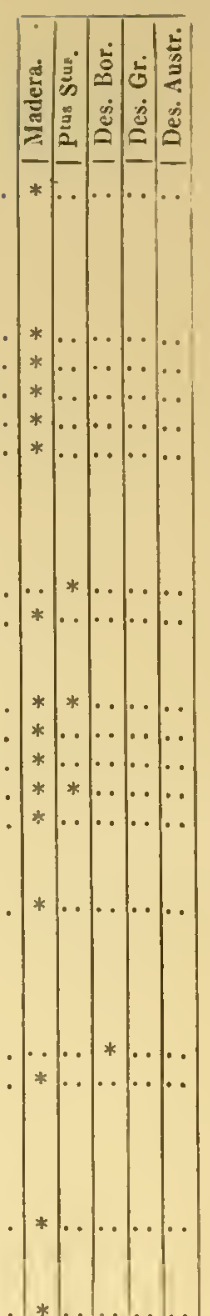



